\title{
Research Paper \\ Guide to Social Work Intervention for Community Re-entry of Offenders With a History of Violence Against Individuals
}

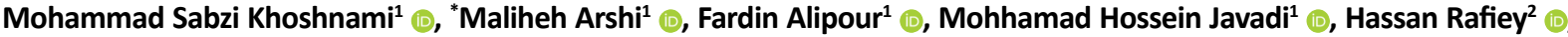 \\ Sara Noruzi ${ }^{3}$
}

1. Department of Social Work, Social Welfare Management Research Center, University of Social Welfare and Rehabilitation Sciences, Tehran, Iran. 2. Department of Social Welfare, Social Welfare Management Research Center, University of Social Welfare and Rehabilitation Sciences, Tehran, Iran. 3. Department of Social Work, Shahid Chamran Hospital, Lorestan University of Medical Sciences, Borujerd, Iran.

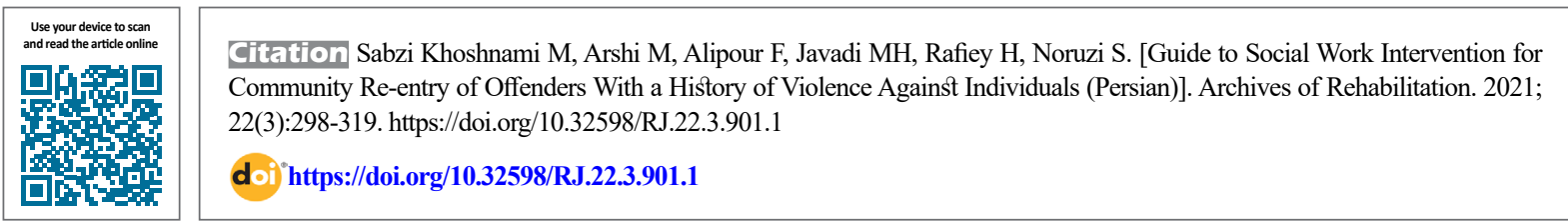

(c) (i) (5)

Received: 28 Aug 2020

Accepted: 24 Feb 2021

Available Online: 01 Oct 2021
Keywords:

Re- entry,

Social rehabilitation,

Violence against individuals, Social work intervention

\section{A B STRACT}

Objective The re-entry of offenders to the community after their imprisonment is a sensitive and important stage and should always be considered by governmental and non-governmental organizations. The proper transition from prison to the community occurs when rehabilitation and social reintegration programs are developed and implemented based on the real needs of individuals. Social work is one of the professions that can help the judicial system with this issue. Many graduates of this profession provide social work services to prisoners and their families. Therefore, it is necessary to identify effective operational solutions in accordance with the conditions of the country and formulate a specific framework. This study aims to prepare a guideline for specialized social work intervention for community re-entry of offenders with a history of violence against individuals. Materials \& Methods In this study, a qualitative approach was used to prepare the specialized guideline. First, the initial barriers and facilitators of successful community re-entry for offenders with a history of violence against individuals were prepared using thematic analysis. Then, based on the protocols of the social work process, the initial draft was developed. Next, at a focus group discussion session with faculty members and experts, the draft was reviewed and improved. To assess its applicability, we used the Appraisal of Guidelines for Research and Evaluation (AGREE) tool.

Results Intertwined concerns, protective shields, welfare/economic security, social support, and achieving relative independence were identified as the five dimensions of successful community re-entry. The specialized social work interventions were presented after approval in four main stages: preparation for the case plan, development of the case plan, interventions and actions, and monitoring and review of interventions. The results of evaluation by AGREE tool showed an overall acceptable average of $7.85 \%$ ( $82.9 \%$ for scope and purpose, $\mathbf{8 8 . 1 \%}$ for stakeholder involvement, $85.6 \%$ for rigor of development, $83 \%$ for clarity of presentation, $84.9 \%$ for applicability, and $89.8 \%$ for editorial independence). The users of this guideline are social workers and other professions related to prisons and training and security organizations, especially after-release care centers. Conclusion Investing in rehabilitation programs for prisoners is one of the best and most cost-effective ways to prevent the reoccurrence of violence, which not only has beneficial effects on those concerned but also promotes public safety. This issue becomes important when social reintegration and rehabilitation programs are developed based on scientific approaches and perspectives. Future studies on the effectiveness of the developed guideline, considering the local conditions, can help identify the effective factors of re-entry to the community in newly released prisoners. The skills and capabilities of social workers in presenting specialized interventions and establishing professional and inter-organizational communication, supporting rules and programs, motivation, and enthusiasm of the offender to change can all contribute to the success of this guideline in achieving the defined goals.

\section{Corresponding Author: \\ Maliheh Arshi, PhD.}

Address: Department of Social Work, Social Welfare Management Research Center, University of Social Welfare and Rehabilitation Sciences, Tehran, Iran Tel: +98 (912) 7397889

E-Mail: barbodalipour@gmail.com; barbodalipour@gmail.com 


\section{English Version}

\section{Introduction}

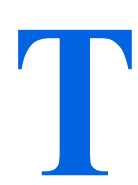

he mental, social, economic, and cultural health of a society is closely related to its security. A large part of this sense of security depends on preventing crime and reducing its recurrence [1]. Violence against individuals constitutes a group of crimes that have increased recently [2]. According to the statistical yearbook of Iran in 2018, personal conflicts and strife have tripled from 247869 cases in 2006 to 753974 cases in 2018 [3]. Focusing on the post- imprisonment period is an essential strategy for social reintegration [4]. According to numerous studies, returning to society after the end of imprisonment is difficult for all people. It is accompanied by various problems such as unemployment, restrictions on communication with family and friends, financial instability, drug use, etc. These problems are compounded when a person is convicted of violence $[5,6]$. O'Brien has conducted numerous studies on the issues and problems of women after imprisonment between 2001 and 2008. These studies have focused on the issues and problems of this period from the perspective of social work. She identified five categories of barriers in the areas of family support, housing, domestic violence, drug/alcohol abuse treatment, employment and economic well-being. According to her, removing these barriers has an influential role in rebuilding life after imprisonment [7].

The proper transfer of people from prison to society occurs when social rehabilitation and reintegration programs are developed and implemented based on their real needs. The output of this type of transfer increases the quality of life and reduces the recurrence of crime [8-11]. The increasing tendency to community re-entry of prisoners over the past decade is due to two intertwined issues. First, policymakers know that failure to respond to the needs of individuals after imprisonment endangers social reintegration and public safety. Second, the interventions presented to offenders are effective for a limited number of them in reducing re-incarceration [12].

Most studies on the social reintegration of people with a history of imprisonment have been conducted in the United States and European countries. In Iran, less attention has been paid to the social issues and challenges of post-imprisonment life [7]. Furthermore, no protocol or guideline for social interventions of people released from prison is found in Iran. Neglecting this issue can increase the recurrence of crime and return to prison, as well as the spread of social harm [13]. Therefore, this study aims to develop a specialized social work intervention guideline to create appropriate conditions for successful community re-entry of offenders after release from prison.

\section{Materials and Methods}

In this study, to prepare a specialized social work intervention guideline, a qualitative method was used in the following seven steps:

Identifying the dimensions of successful community reentry and categorizing them (output of the qualitative section): In this step, 26 semi-structured interviews with individuals with a history of violence and with experts were conducted in 2019. For data analysis, we used Brown and Clarke's (2006) six-step thematic analysis procedure [14].

Review of studies to prepare a matrix of dimensions of successful community re-entry (output of literature review): A search was conducted in the national and international databases on quantitative studies and protocols and guidelines for the community re-entry using MeSH keywords of "successful re-entry", "re-entry", "social reintegration", "return to the community", "re-entry successful".

Preparing an initial pool of barriers and facilitators to successful community re-entry by combining two qualitative and literature review sections based on the emic (qualitative) and etic (literature review) approach of Whittemore and Knafl (2005). We used these tools to analyze and recognize the dimensions of successful community re-entry [15]. Also, thematic synthesis made it possible to identify barriers and facilitators besides similarities and differences [16].

Drafting the specialized intervention guideline: In this stage, an initial draft of the guideline was prepared by the research team based on the previous findings, compliance with existing protocols, and receiving feedback from experts. Five guidelines of "Handbook on the Prevention of Recidivism and the Social Reintegration of Offenders, United Nations Office on Drugs and Crime (UNODC) 2018" [17], "Guide to the rehabilitation and social reintegration of women prisoners: Implementation of the Bangkok Rules (2019)" [18], "Mapping Prisoner Reentry: An Action Research Guidebook [19]", "Effective Case Management (implementation of effective practices that will support successful offender outcomes)" [20], and "the World Health Organization handbook for guideline development [21]" were used as a basis for drafting the specialized intervention guideline in this study. Review and development of the drafted guideline: It was done at a Focus Group Discussion (FGD) session. 
Table 1. Social work intervention guideline based on case management approach

\begin{tabular}{|c|c|c|}
\hline Steps & Interventions and Tools & Achievements \\
\hline $\begin{array}{l}\text { Preparation for the case plan: This step } \\
\text { involves identifying people in need of } \\
\text { services and their initial and complimentary } \\
\text { assessments focusing on identifying } \\
\text { potential risks, needs, and strengths or } \\
\text { abilities (from admission to achieving the } \\
\text { goals). }\end{array}$ & $\begin{array}{l}\text { Acceptance, professional and empathetic } \\
\text { communication, interview, observation, } \\
\text { genealogy drawing, ecological mapping, } \\
\text { descriptive, participatory, competency- } \\
\text { based assessment, and home visit }\end{array}$ & $\begin{array}{l}\text { Declare of readiness to start cooperation, } \\
\text { persuaded to start the work process, } \\
\text { accurate identification of issues and } \\
\text { problems of clients, categorization } \\
\text { of problems, paying attention to the } \\
\text { capabilities and capacities of clients }\end{array}$ \\
\hline $\begin{array}{l}\text { Development of the case plan: This step } \\
\text { involves targeting and planning. }\end{array}$ & $\begin{array}{l}\text { Agree on general goals, goals being SMART } \\
\text { (specific, measurable, achievable, relevant, } \\
\text { and time-bound), one-on-one meetings } \\
\text { with clients and group meetings with } \\
\text { other specialists such as psychiatrists, } \\
\text { psychologists, legal and occupational } \\
\text { experts, and head of the center regarding } \\
\text { planning to support and organize clients }\end{array}$ & $\begin{array}{l}\text { Participation of clients in designing goals, } \\
\text { coordination of case plan activities with } \\
\text { interventions that meet the specific needs } \\
\text { of clients, formulation of specific, tangible, } \\
\text { easy, and trackable plans }\end{array}$ \\
\hline \multirow{6}{*}{$\begin{array}{l}\text { Intervention and action: This step includes } \\
\text { specialized social work interventions. }\end{array}$} & $\begin{array}{l}\text { Psychosocial, social, economic, civic } \\
\text { supports (to assess progress, social } \\
\text { support questionnaires developed by } \\
\text { Sarason, Phillips, Norbeck, and Wax were } \\
\text { recommended) and case management } \\
\text { referral form }\end{array}$ & $\begin{array}{l}\text { Identification and evaluation of support } \\
\text { resources, linking between authorities and } \\
\text { social resources, facilitation }\end{array}$ \\
\hline & $\begin{array}{l}\text { Counseling and training: In accordance } \\
\text { with family, occupational, educational } \\
\text { counseling protocols, counseling and } \\
\text { guidance to encourage the treatment of } \\
\text { physical/mental diseases and substance } \\
\text { abuse, training in communication skills, } \\
\text { especially anger management }\end{array}$ & $\begin{array}{c}\text { Improvement and promotion of } \\
\text { performance based on the type of } \\
\text { counseling, and awareness, informing and } \\
\text { increasing the knowledge and capability of } \\
\text { accessible clients based on the educational } \\
\text { program }\end{array}$ \\
\hline & $\begin{array}{l}\text { Establishing a relationship between } \\
\text { the personal and social resources and } \\
\text { maintaining and strengthening positive } \\
\text { relationships (to assess progress, the use } \\
\text { of communication skills questionnaires } \\
\text { developed by Queen Dam, Jarabak, and } \\
\text { Barton were recommended) }\end{array}$ & $\begin{array}{l}\text { Improving communication and } \\
\text { interpersonal skills and increasing the } \\
\text { usability of social resources for reducing } \\
\text { violent behaviors and managing behavior }\end{array}$ \\
\hline & $\begin{array}{l}\text { Group interventions and family work: } \\
\text { in accordance with the standards of } \\
\text { group social work (use of training groups, } \\
\text { discussion, and exchange of ideas, peer } \\
\text { groups to exchange positive experiences } \\
\text { in anger management, growth and } \\
\text { development, therapy, and counseling, } \\
\text { forgiveness-oriented groups) }\end{array}$ & $\begin{array}{l}\text { Improving family skills and relieving } \\
\text { tensions, performing group tasks, and } \\
\text { enjoying the benefits of group dynamics }\end{array}$ \\
\hline & $\begin{array}{l}\text { Community interventions and social } \\
\text { facilitation: in accordance with } \\
\text { the standards of social work (local } \\
\text { development, planning, and social action), } \\
\text { use of extensive social education aimed at } \\
\text { improving social skills, continuous social } \\
\text { monitoring, comprehensive services with a } \\
\text { focus on support }\end{array}$ & $\begin{array}{l}\text { Reducing social barriers, de-stigmatization, } \\
\text { seeking support, and supporting }\end{array}$ \\
\hline & $\begin{array}{l}\text { Monitoring and follow-up: planning based } \\
\text { on each case by phone, email, or in-person }\end{array}$ & $\begin{array}{l}\text { Taking care of the gains achieved during } \\
\text { the intervention, ensuring that the case } \\
\text { has access to resources, identifying new } \\
\text { problems, and trying to solve them }\end{array}$ \\
\hline
\end{tabular}




\begin{tabular}{|c|c|c|}
\hline Steps & Interventions and Tools & Achievements \\
\hline $\begin{array}{l}\text { Monitoring and reviewing the intervention: } \\
\text { This step includes reviewing the previous } \\
\text { steps and monitoring the achievement } \\
\text { of the defined goals, and, if necessary, } \\
\text { reviewing the interventions. }\end{array}$ & $\begin{array}{c}\text { Case management checklist, self-report } \\
\text { tool, monitoring reports, and related } \\
\text { questionnaires }\end{array}$ & $\begin{array}{l}\text { The social worker can use the defined } \\
\text { goals to evaluate the effectiveness of } \\
\text { the interventions. Goals are assessed } \\
\text { quantitatively and qualitatively. Quantitative } \\
\text { goals are such goals as suitable housing, } \\
\text { finding a job with sufficient income, and } \\
\text { gaining the desired job or technical skills, } \\
\text { and qualitative goals are such goals as the } \\
\text { feeling of individual and family satisfaction, } \\
\text { participation in society, reducing criminal/ } \\
\text { violent behaviors, increasing the level } \\
\text { of resilience in challenging situations, } \\
\text { achieving relative independence, improving } \\
\text { and correcting social and interpersonal } \\
\text { behaviors which are related to more } \\
\text { complicated interventions and require } \\
\text { tools and standards for assessment. The } \\
\text { maximum achievement of the defined goals } \\
\text { is considered. }\end{array}$ \\
\hline
\end{tabular}

Rehabilitation

Assessing the applicability of the designed guideline: It was done using the Appraisal of Guidelines for Research And Evaluation (AGREE) tool [22]. The average total report of 10 experts for six domains was $85.7 \%$, indicating that all experts approved the applicability of the guideline. Modifying based on suggestions and criticisms and finalizing the intervention guideline.

\section{Results}

According to the experts and the literature based on the case management approach, the guideline draft was compiled by the research team focusing on the information obtained from both qualitative and quantitative parts. Experts in this field were used to review the content in an FGD session. The case management approach is commonly used for caring for people released from prison and those with a history of substance abuse disorders [23]. This approach has empirical scientific support [24]. According to this approach, clients have active participation in providing specialized interventions, and goals are achieved by relying on the interventions provided by social workers and client participation. Therefore, the guideline of specialized social work intervention for community re-entry of offenders based on the case management approach was presented in four main stages: 1) preparation for the case plan, 2) development of the case plan, 3) intervention and action, 4) monitoring and reviewing the intervention (Table 1).

\section{Discussion and Conclusion}

Research shows that in the case management approach, adapting the severity of interventions to the assessed level of risk (e.g. more intensive strategies for high-risk offenders) leads to better outcomes [25]. Throughout the evaluation and intervention planning process, case managers act as a bridge between internal/external resources and individuals to achieve positive change. This bridge seeks to increase prisoners' motivation and acquire clear cooperation between providers and recipients of services and continued care for people released from prison so that a successful community re-entry can finally be achieved [23].

The "Guide to the rehabilitation and social reintegration of women prisoners: Implementation of the Bangkok Rules" [18] is consistent with our guideline. This guideline identifies the various aspects of successful rehabilitation of female prisoners. The main focus is on specific programs and appropriate measures to address the underlying causes of the crime. This guideline covers a wide range of programs, including medical care, mental health care, substance abuse prevention and treatment programs, counseling, psychosocial support, individual and group training courses, employment and vocational training, housing, and more.

Several studies have shown that improving individuals' psychosocial abilities, such as skills training in challenging situations and correcting communication skills, have significantly prevented the recurrence of violence against individuals [26-28]. In the present study, the use of educational and counseling programs (individual and family) was considered in the case management program as one of the 
measures to overcome stressful situations. According to the findings, one of the barriers to community re-entry was stigmatization and rejection by others. Mirhosseini and Larijani (2018) and Kafashi and Eslami (2009) concluded that to prevent the recurrence of crime. For community re-entry of offenders, it is necessary to remove formal and informal labels applied by institutions, friends, family, and society. In the present study, by using social work methods, planning was done to identify these labels and inappropriate environments. Studies by Sutherland, Durkheim, and Cohen have also shown that inappropriate living environments are factors in the emergence and recurrence of crime. Individuals learn criminal behaviors by interacting with groups that carry criminal norms. Labeling causes social alienation, job loss, and leaving family and friends [29]. Therefore, the use of local development methods, social planning, and social action in identifying inappropriate environments, labeling factors, and implementing the necessary interventions in this guideline was recommended. Hezar Jaribi believed that the expansion of Non-Governmental Organizations (NGOs) in the form of neighborhood-based and facilitation offices could effectively control social harms comprehensively [30].

Another area highlighted in the guideline in this study was social support with a focus on family. Some studies have shown that the more people are supported and controlled by their family members after release from prison, the less likely they will commit a crime again. For this reason, one of the ways to re-enter the community is to be with family. In this regard, in the present study, group methods and attracting the participation and support of families in improving the situation of the target group were used. In addition to increasing the likelihood of community re-entry, familycentered interventions reduce aggressive behaviors and deviance in children [30]. Other studies have mentioned social support [31], supervision and interaction of family members during and after imprisonment, and socializing with friends [32-34].

An essential strategy in intervention guides and programs with a case management approach is to seek support from NGOs, charity centers, and other civic associations, given the limitations of government resources [35]. For this reason, in the present guideline, social workers are required to provide a variety of external and internal support for the target group referring to these centers. Support seeking is part of the specialized interventions of social workers that are done with the participation and cooperation of clients. Its effectiveness has been shown in various studies [36,37]. They also use referral techniques to link the individual to the support resources. Using facilitation methods, social workers provide access to social resources and identify administrative barriers to service delivery. There is a relation- ship between all the mentioned interventions that lead to achieving the defined goals [38]. In this way, social workers, by various methods and techniques and using the participation, capacity, and capabilities of the individuals and their families, design a unique intervention program for them that can lead to their empowerment.

The outcome of this study was a guideline for social work intervention based on a case management approach for successful community re-entry and reduction of problems related to social rehabilitation. Policymakers and social workers as service providers in post-release care centers are recommended to apply the recommendations in this guideline for the social integration of individuals after the end of imprisonment. Future studies on the effectiveness of this specialized social work intervention guideline, considering local conditions, can help identify the influential factors of community re-entry of people released from prison, especially those convicted of violence against individuals.

\section{Ethical Considerations}

\section{Compliance with ethical guidelines}

This study was approved by the Ethics Committee of University of Social Welfare and Rehabilitation Sciences (Code: IR.USWR.REC.1399.180).

\section{Funding}

This article is part of the findings of the $\mathrm{PhD}$. dissertation of the first author at the Department of Social Work, University of Social Welfare Sciences and Rehabilitation.

\section{Authors' contributions}

All authors equally contributed to preparing this article.

\section{Conflict of interest}

The authors declared no conflict of interest.

\section{Acknowledgments}

The authors would like to thank the General Directorate of Prisons of Tehran Province and the Center for Social Services and Post-Exit Care of Tehran Province. 
راهنماى مداخله تخصصى مددكارى اجتماعى در فرايند بازگمثت به جامعه محكومين با سابقه خشونت

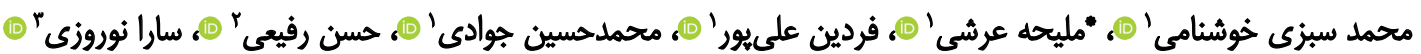

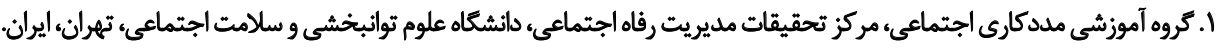

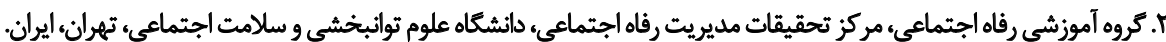

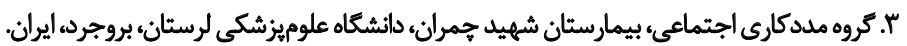

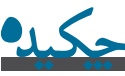

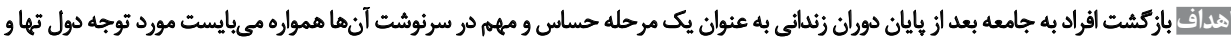

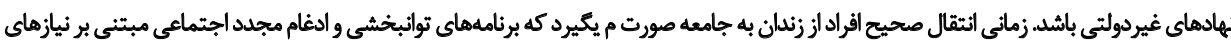

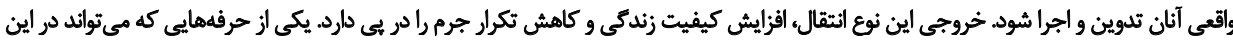

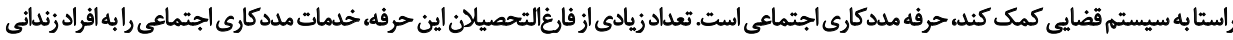

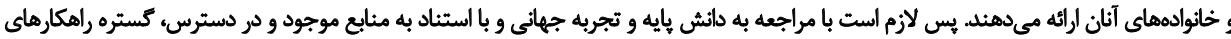

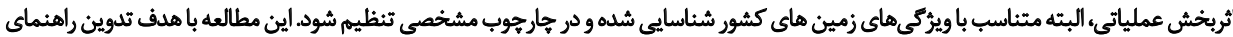

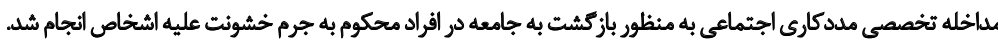

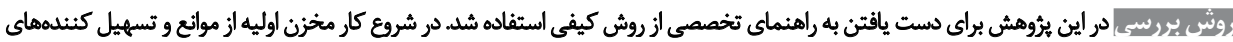

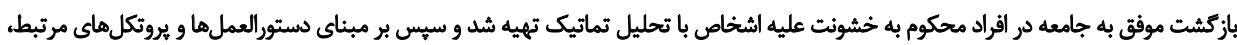

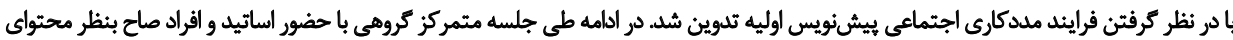

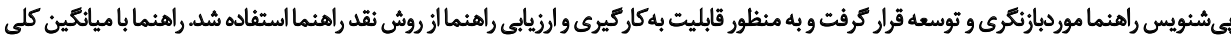

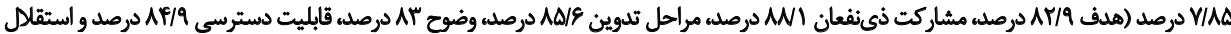

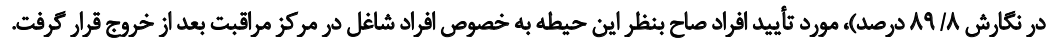

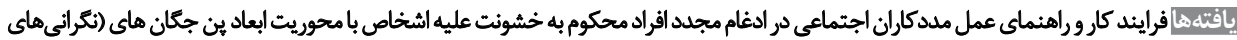

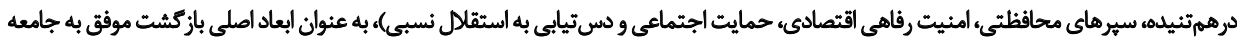

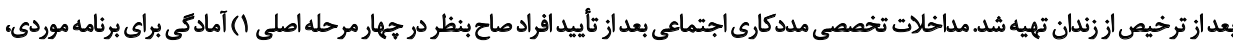

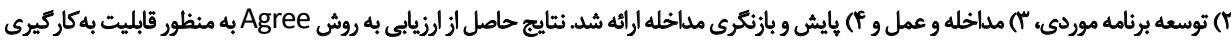

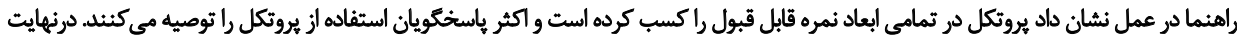

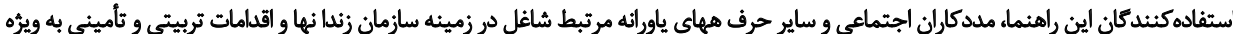
مركز مراقبت بعد ازخ خروج هستيند.

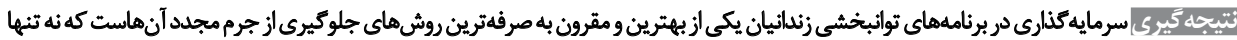

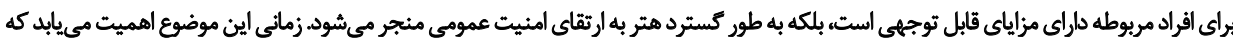

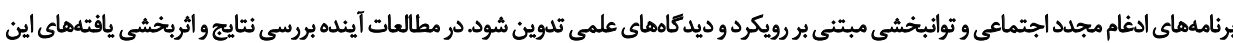

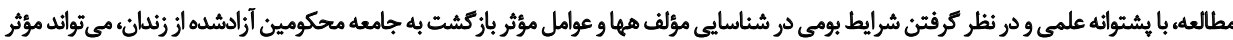

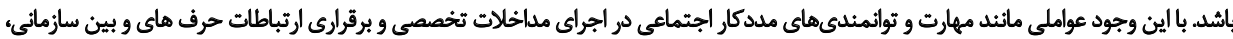

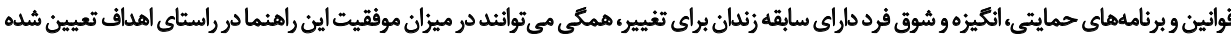

تاريخ دريافت: V• شهريور و

تاريخ يذيرش: 9. اسفند

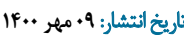

كليدوأوهان

باززكشت به جامعه،

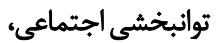
خشونت عليه اشخاص، افراد زنداني، راهنماى الشخاص، مددكارى اجتماعى.

-

" دويسنده مسئول:

دكتر مليحه عرشى : نواينده

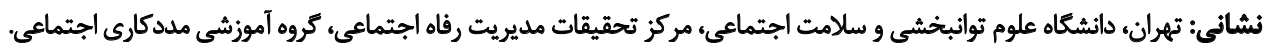

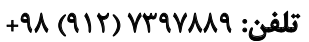

رايانامه: 


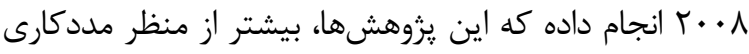

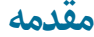

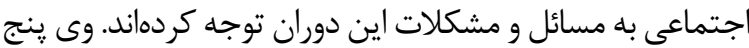

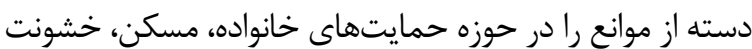

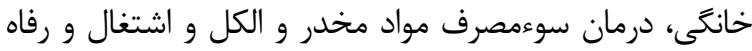

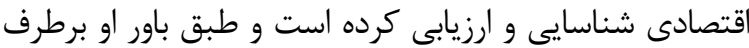

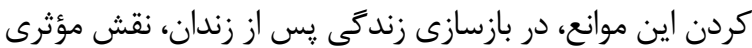

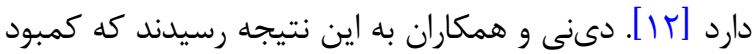

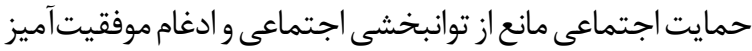

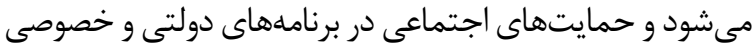

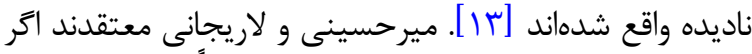

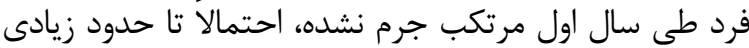

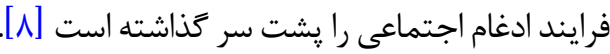
زمانى انتقال صحيح افراد از زندان به جامعه صورت مي گئيرد كه

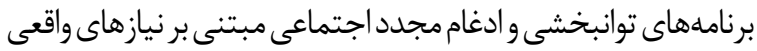

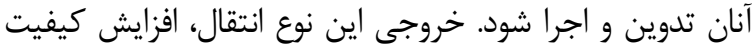

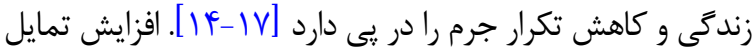

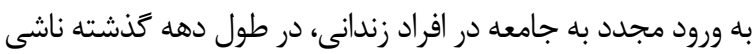

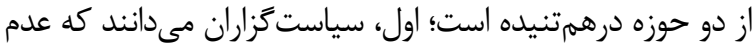

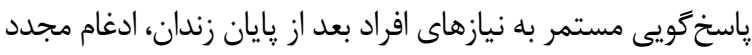

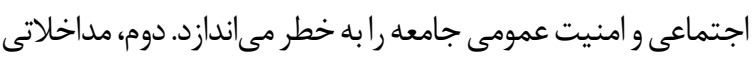

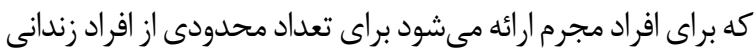

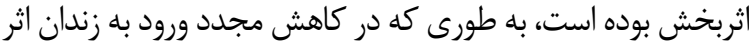

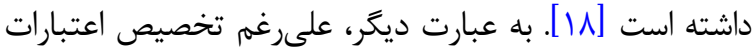

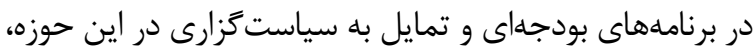

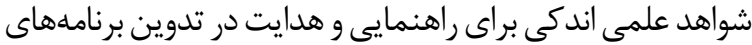

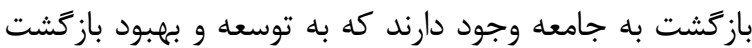

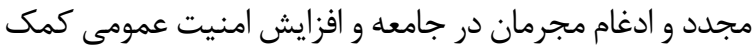

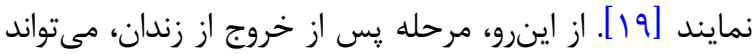

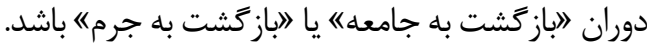

يكى از حرفههايى كه ميتواند در اين راستا به سيستم

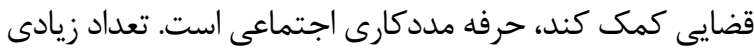

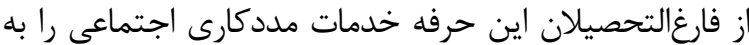

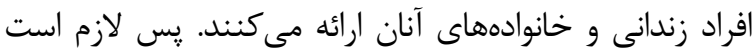

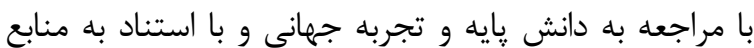

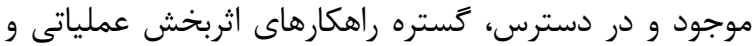

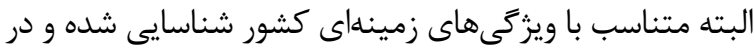

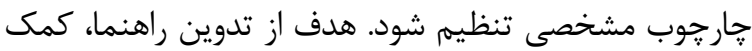

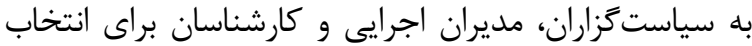

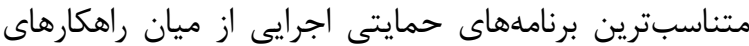

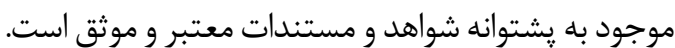
بر اساس مرور منابع، اكثر مطالعات در خصوص ادغام إندام مجدد

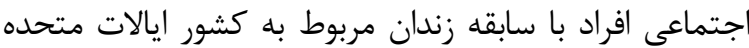

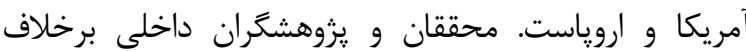

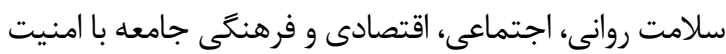

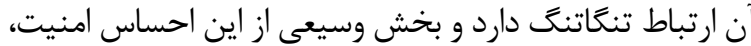

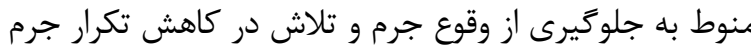

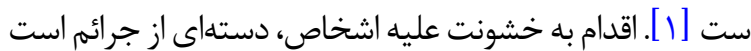

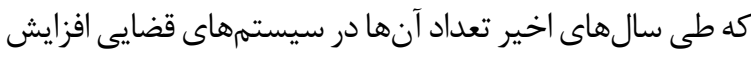

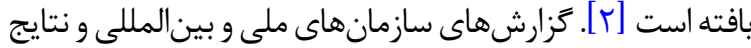

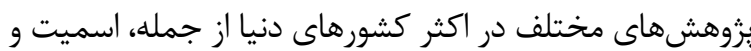

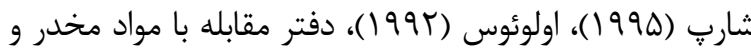

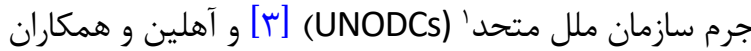

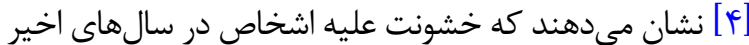

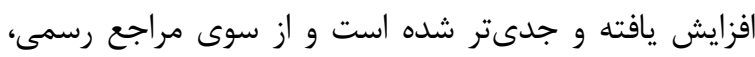

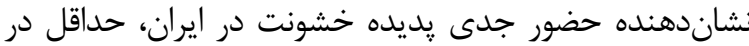

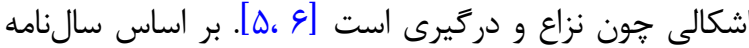

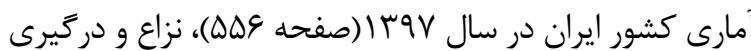

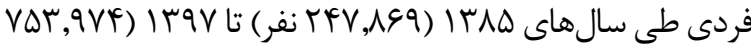

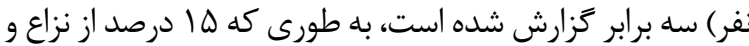

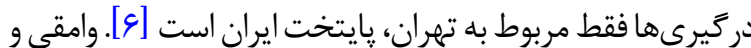

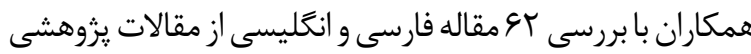

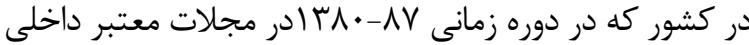

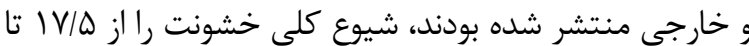

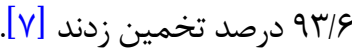

تمركز بر دوران بعد از حبس، يك راهكار مهرم براى ادغام إمبام

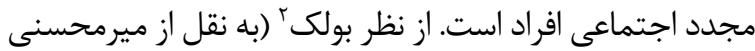

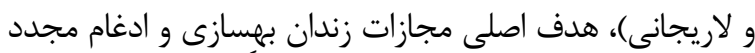

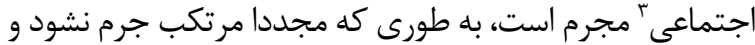

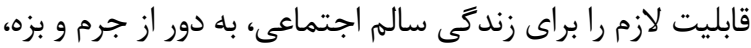

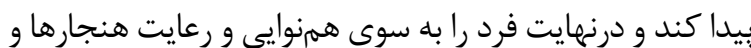

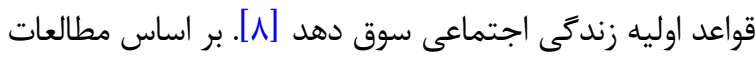

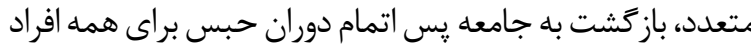

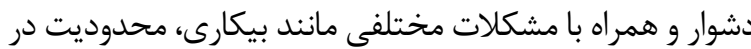

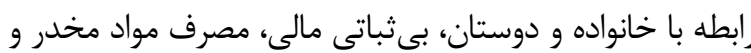

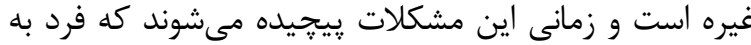

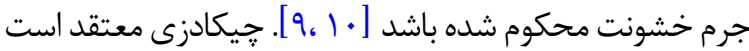

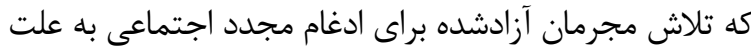

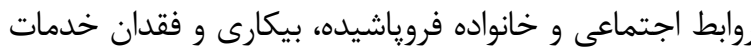

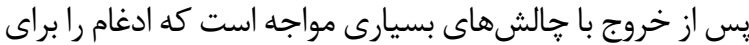

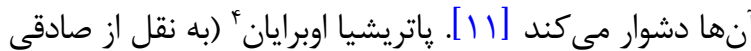

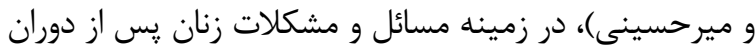

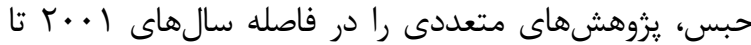

\section{United Nations Office on Drugs and Crime (UNODCs)}

3. Social reintegration

4. O'Brien 
جرم خشونت عليه اشخاص انجام شد و با استناد به يافتههاى

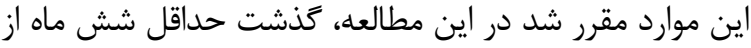

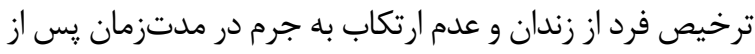

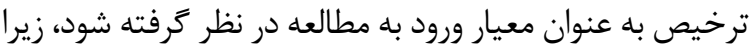
در اين مدت فرد تا حدودى به شرايط پِايدار رسيده است.

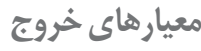

- فرد مايل به شركت در يزوهش نباشد.

- درصورتى كه فرد در طول يزوهش هريك از معيارهاى ورود

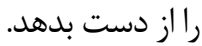

يزوهشكَر با معرفىنامه دانشگاه علوم توانبخشى و سلامت

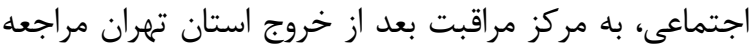

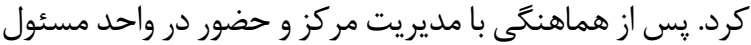

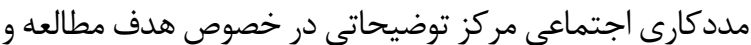

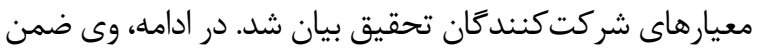

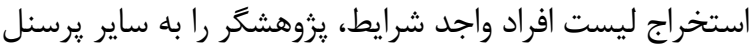

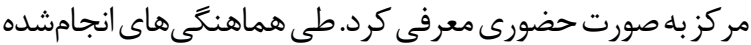

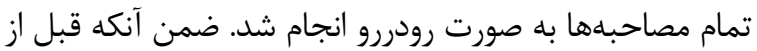

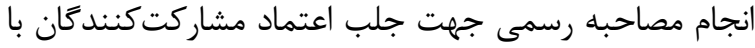

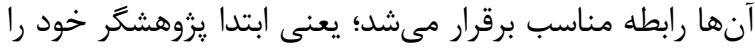

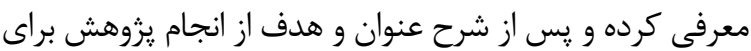

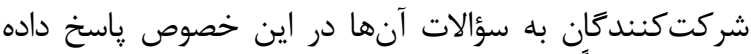

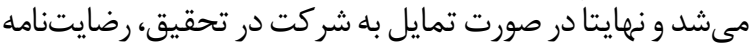

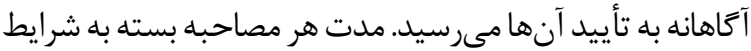

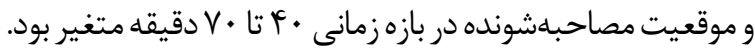

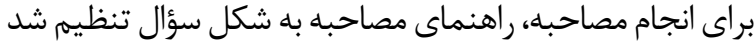

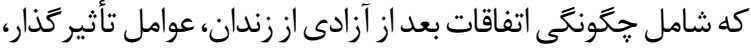

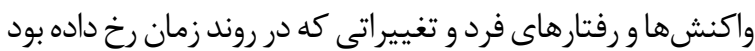

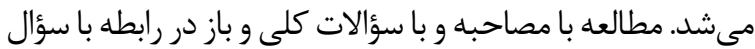

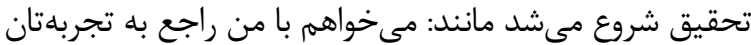

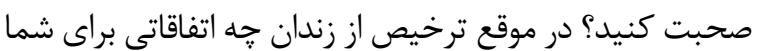

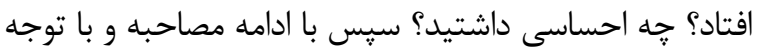

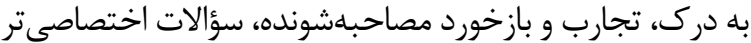

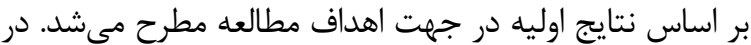

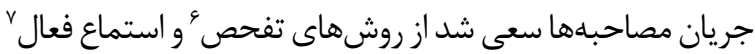

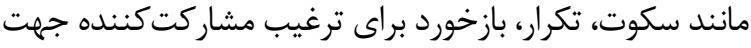

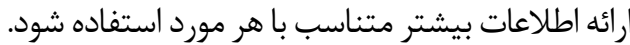

\section{نمونه سؤالهاى مربوط به شركت كنند}

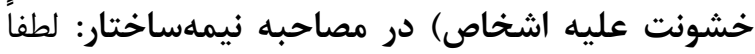

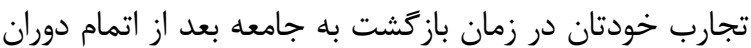

\section{Probing}

7. Active listening

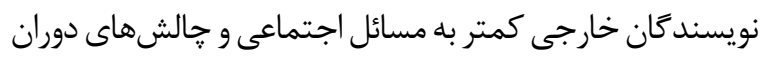

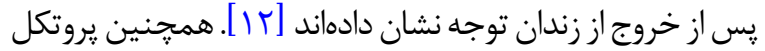

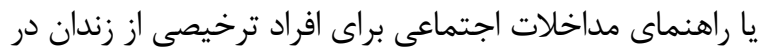

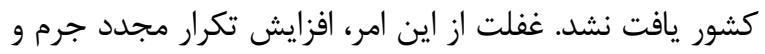

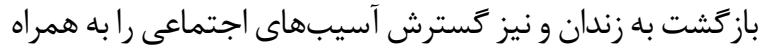
دارد [بار]

بنابراين هدف از اين مطالعه، تدوين راهنماى مداخله تخصصى

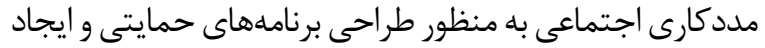

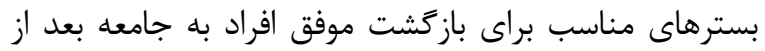

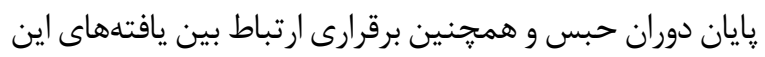

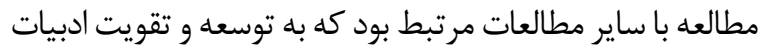

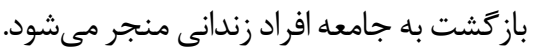

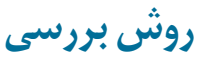

در اين يزوهش براى دست يافتن به راهنماى تخصصى، از

$$
\text { روش كيفى و طى هفت مرحله زير استفاده شد: }
$$

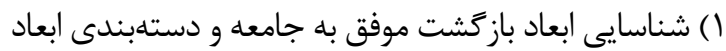

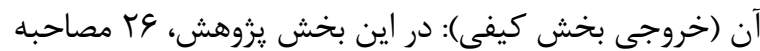

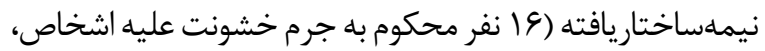

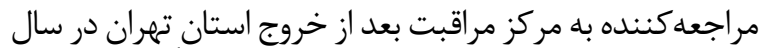

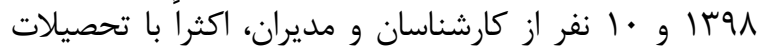

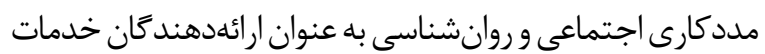

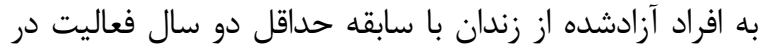

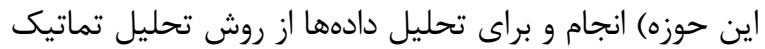

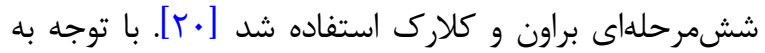

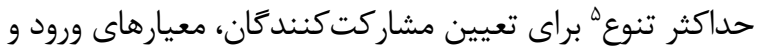
خروج به شرح زير در نظر گرفته شد: تئن

$$
\text { معيار هاى وروود }
$$

- داراى سابقه حبس به دليل ارتكاب جرم خشونت عليه اشخاص (تمام مصاديق اين نوع جرائم) باشند. - حداقل مدت يك سال حبس را در زندان كذرانده باشند

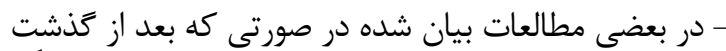

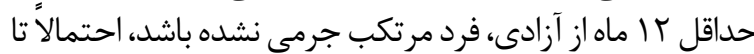

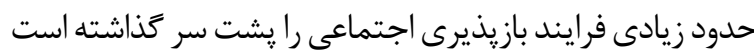

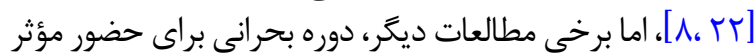

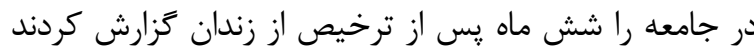

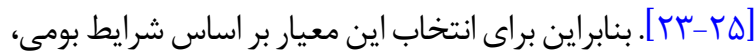

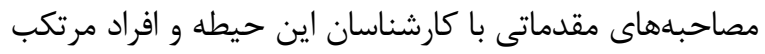




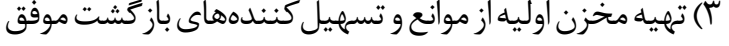

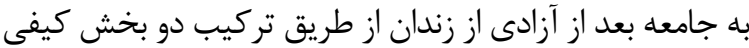

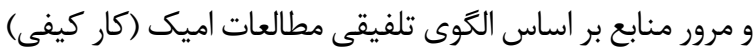

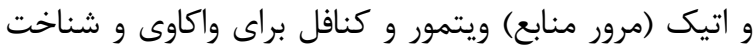

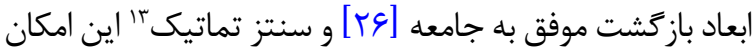

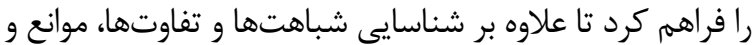

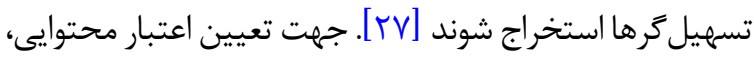

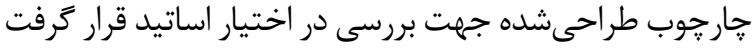

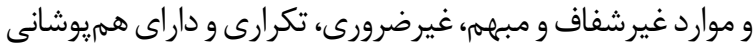
اصلاح شد.

F) تدوين بِيشنويس راهنماى مداخلات تخصصى (توسط

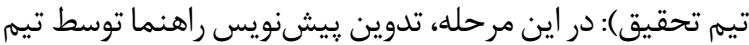

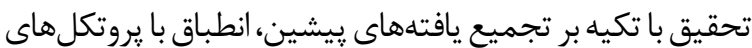

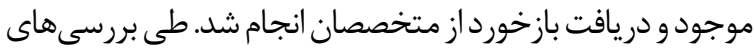

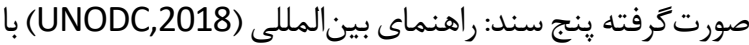

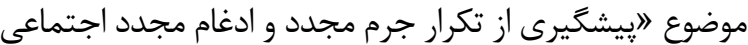

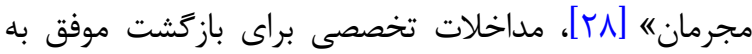

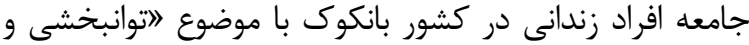

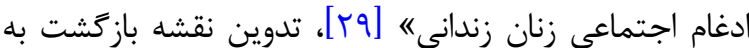

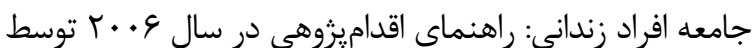

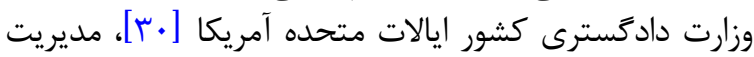

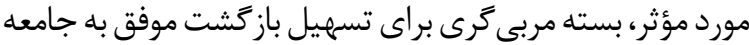

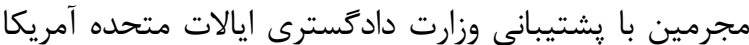

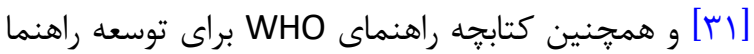

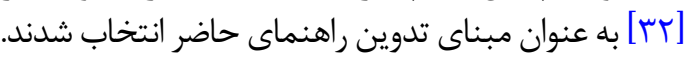

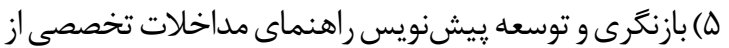

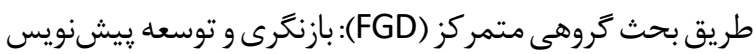

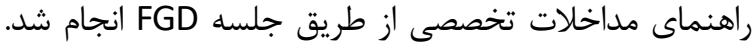

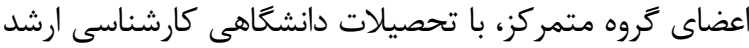

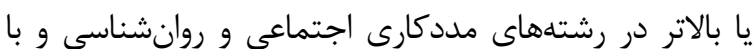

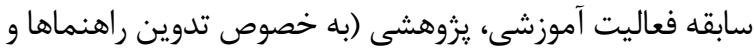

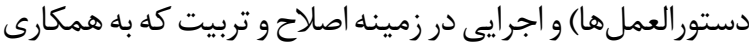

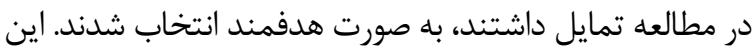

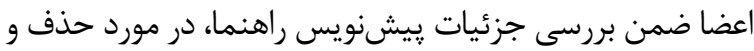

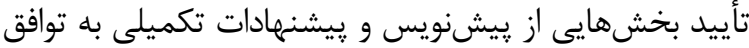

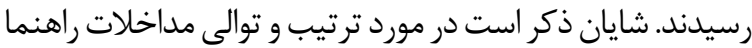

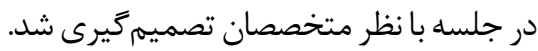

9) بررسى قابليت بهكاركيرى راهنماى مداخلات تخصصى (از

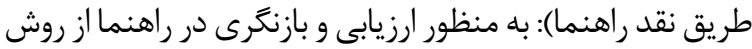

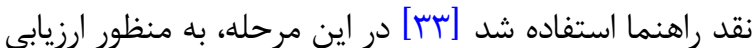
و بازنخرى در راهنماى مذكور از روش نقد راهنما توسط افراد إنداد

\section{Thematic synthesis}

حبس را توضيح دهيد؟ جه اتفاقى برايتان رخ داد و جه شر شرايطى را

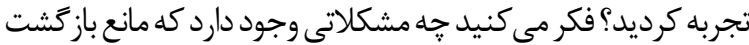

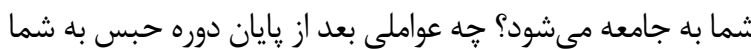

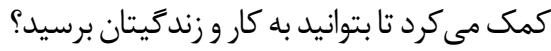

نمونه سؤالهاى مربوط به شركت كنندكان (كارشناسان و

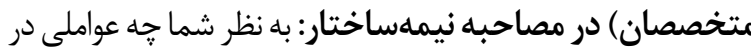

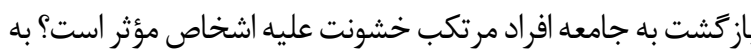

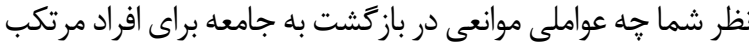

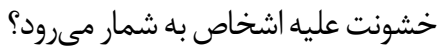

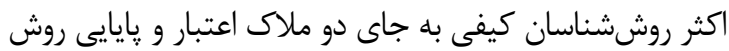

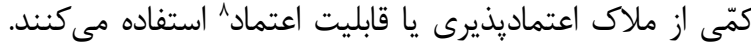

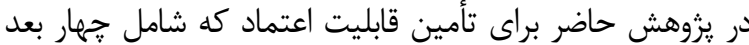

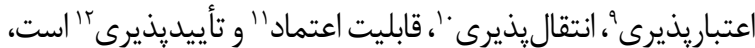

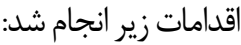

نسبت به انتخاب شركت كنند كان بر اساس معيارهاى نمونه كيرى،

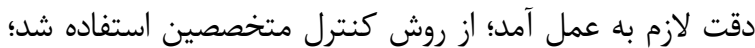

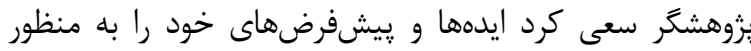

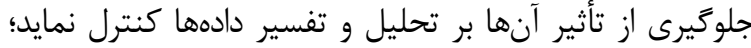

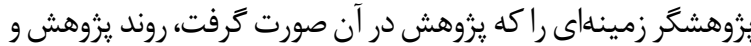

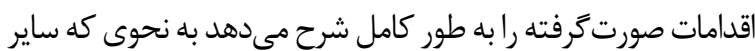

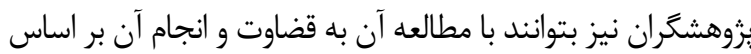

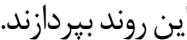

(T) مرور منابع به منظور تهيه ماتريس ابعاد باز گشت موفق به جامعاه

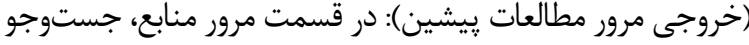

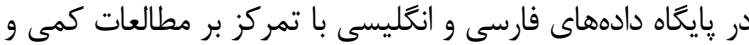

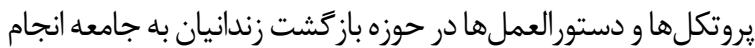

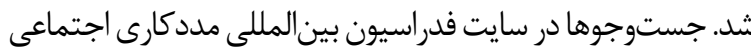

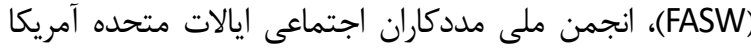

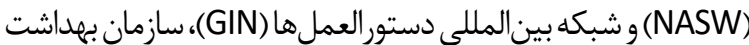

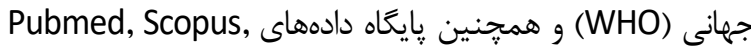
Web of sciences

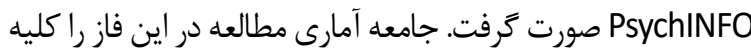

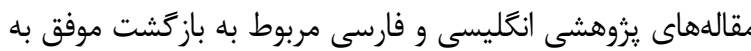

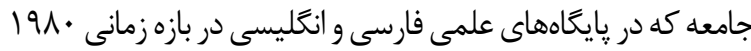

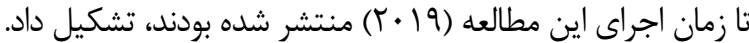

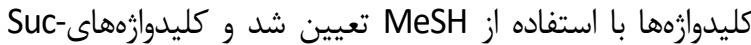
cessful Reentry to community, Reentry to community, Social reintegration, return to community, reentry successful

\section{Trustworthiness or rigor in Qualitative}

9. Credibility

10. Transferability

11. Dependability

12. Confirm ability (Fittingness) 


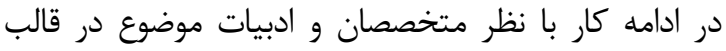

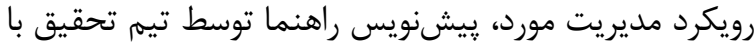

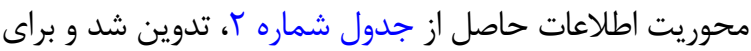

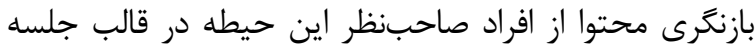

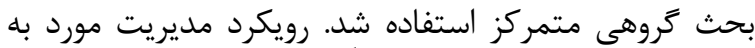

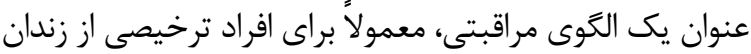

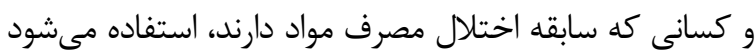

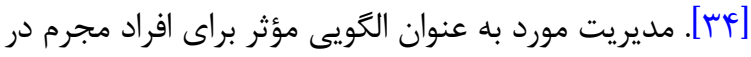

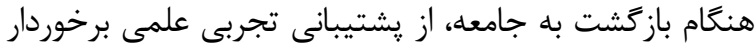

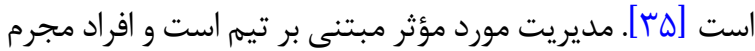

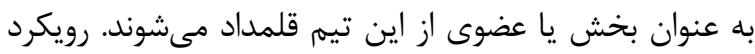

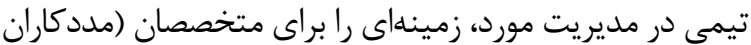

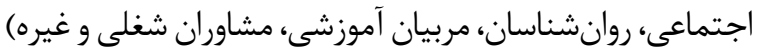

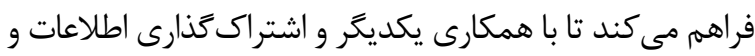

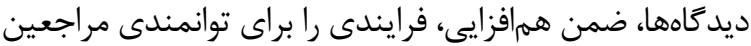

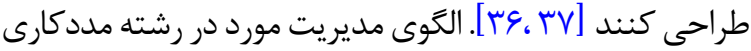

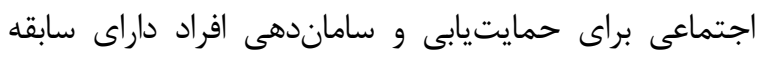

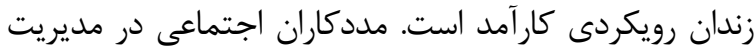

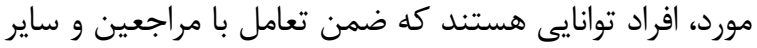

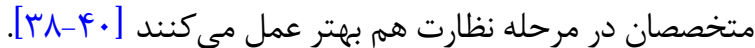

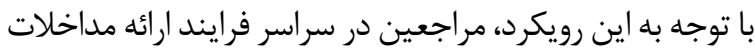

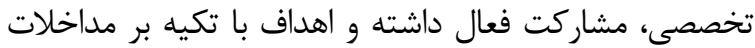

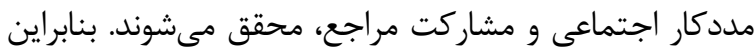

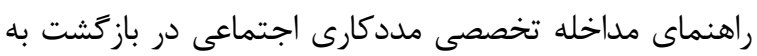

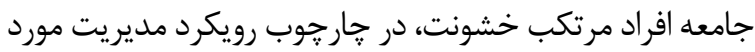

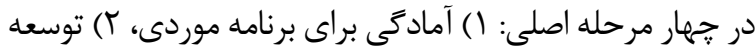

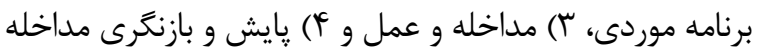

$$
\text { ارائه مىشود (جدول شماره بَ). }
$$

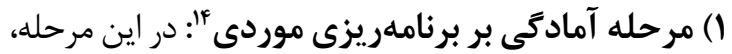

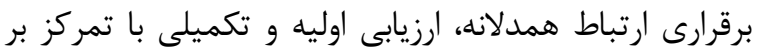

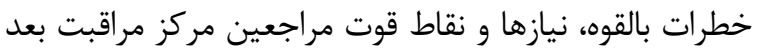

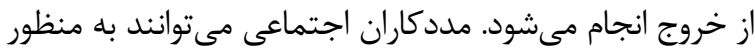

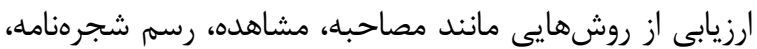

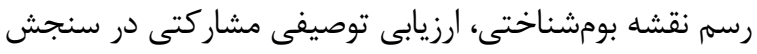

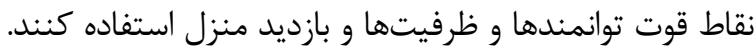

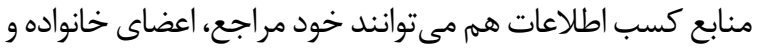

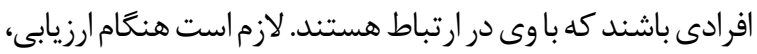

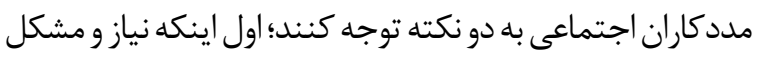

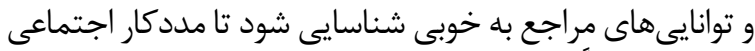

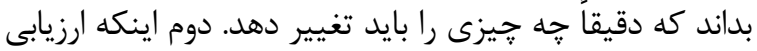

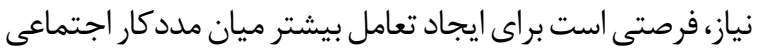

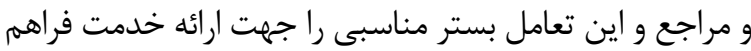

\section{Preparing for case planning}

صاحبنظر بهويزه افراد شاغل در مراكز مراقبت بعد از خروج كه داري

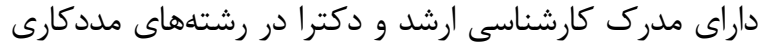

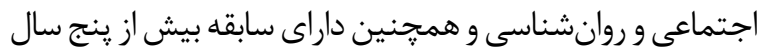

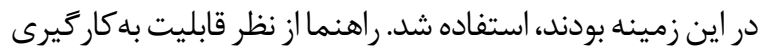

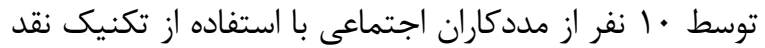

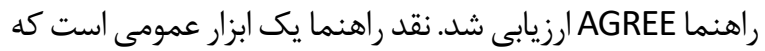

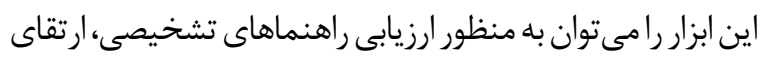

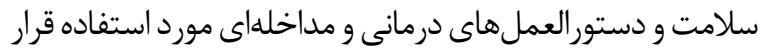

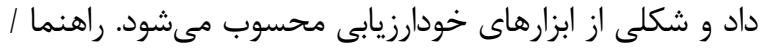

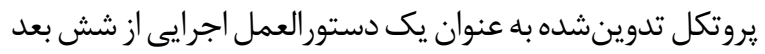

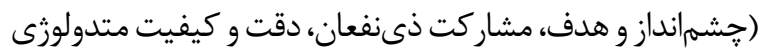

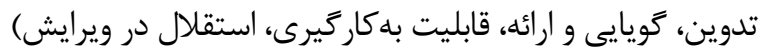

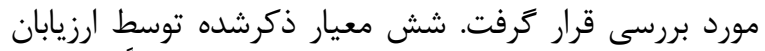

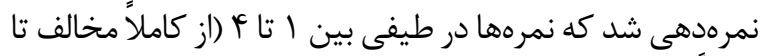

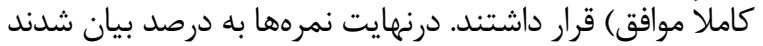
كه نتايج در جدول شماره إ ارائه شده است.

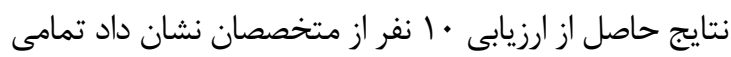

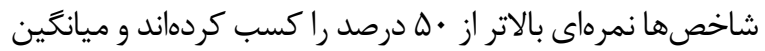

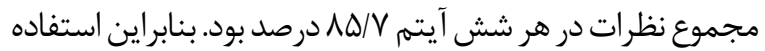
از يروتكل توسط تمامى ارزيابان تأييد شد.

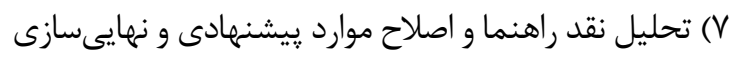

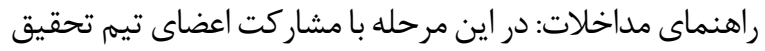

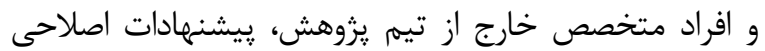

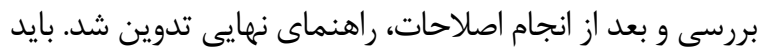

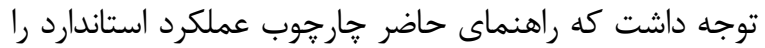

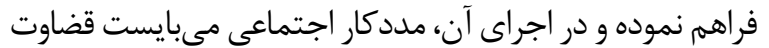

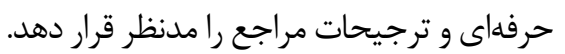

يافتهها

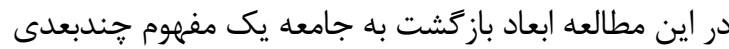

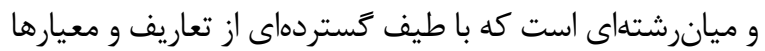

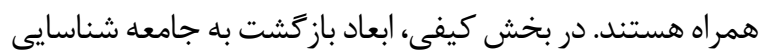

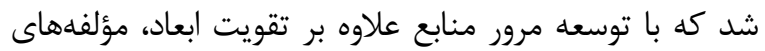

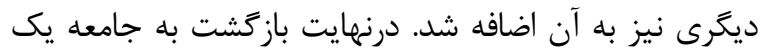

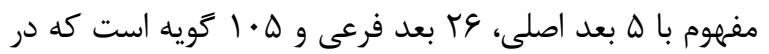

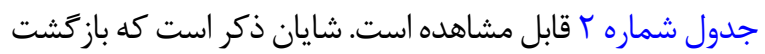

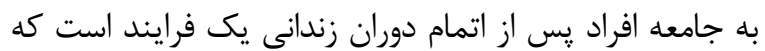

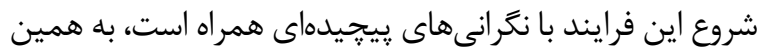

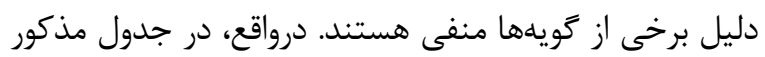

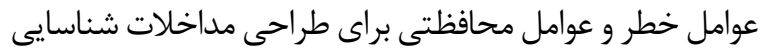

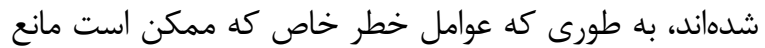

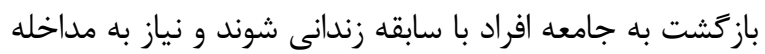
دارند، شناسايى شدهاند. 
جدول ا. يتايج حاصل از اززيابى با روش نقد راهنما

\begin{tabular}{|c|c|c|c|c|c|c|c|}
\hline \multirow{2}{*}{ ارزيابي نهايى } & \multicolumn{6}{|c|}{ ورصد } & \multirow{2}{*}{ ارزيابان } \\
\hline & عدم وابستكي در نكارش & قابليت ديستر سيى & وضوح ارائه & مراحل تدوين & ذئفعان & هدف و محدورده & \\
\hline اكيداً توصيه مي كثم. & 10. & $\Delta \Psi / N$ & $q \Psi \mathrm{N}$ & $\Lambda V / T$ & $9 \Psi / N$ & vo & شماره \\
\hline مشروط به انجام تغييرات توصيه مى كنم. & $1 .$. & $91 / 8$ & Al/ $/ \mathrm{T}$ & $9 Y / \Lambda$ & $\Delta V / \Delta$ & $91 / 8$ & 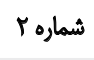 \\
\hline اكيدأ توصيه مي كنه. & 1.. & 1.0 & $1 \cdots$ & $1 \cdots$ & $1 .$. & $1 .+$ & 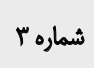 \\
\hline هشروط به انجام تغييرات توصيه مى كنه. & $g \pi / \Delta$ & $\Delta N /$ & $9 \pi / \Delta$ & $n / 4$ & sT/Q & $\Delta N r^{r}$ & Fماره F F F \\
\hline اكيدأ توصيه مىكنم. & $A V / \Delta$ & $1 .$. & vo & $\Lambda A / Y$ & $9 \% / V$ & $1 \cdots$ & شماره ه \\
\hline مشروط به انجام تغييراك توصيه مي كثم. & $1 .$. & १บ/ & $A V / \Delta$ & $n / e$ & 1.0 & $A r / r$ & شماره \& \\
\hline اكيداً توصيه مى كنه. & 1.0 & vo & $9 \Psi / N$ & $9 Y / A$ & $A V / \Delta$ & १พ/8 & شماره \\
\hline اكيداً توصيه مي كثم. & va & $1 \ldots$ & $A v / T$ & $1 \mathrm{~A} / \mathrm{Y}$ & $9 \% / V$ & 10. & Aماره A A \\
\hline مشروط به انجام تغييرات توصيه مى كثم. & $V / \Delta$ & $\Delta N r$ & ET/Q & va & ev & r & شماره 9 \\
\hline اكيداً توصيه مى كنه. & 1.0 & $91 / 8$ & $9 T / Y$ & $9 \Psi / Y$ & $1 .$. & $1 \cdots$ & 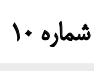 \\
\hline جمع كل نمرهها : N م درصد & 19/A & $1 F / 9$ & Ar & $1 \mathrm{~A} / \mathrm{F}$ & $\mathcal{W I}^{\prime}$ & $\Lambda T / q$ & جمعبندى \\
\hline
\end{tabular}

جنانجه هركدام از طرفين از اين قرارداد كارى تخطى نمايند، نياز

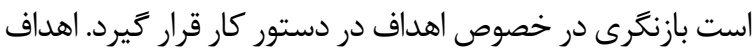

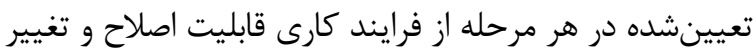

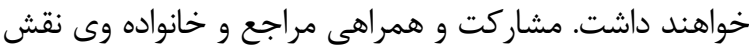

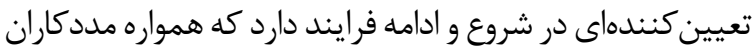

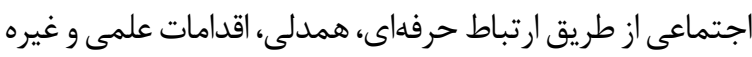

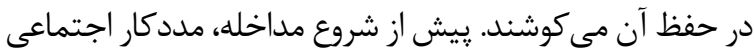

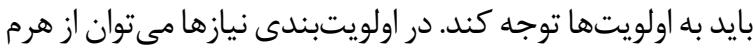

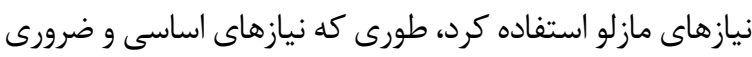

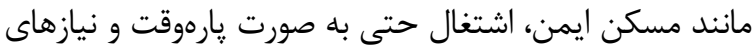
ارتباطى در اولويت بيشترى قرار دارند.

علاوه بر اين، در اين مرحله براى هدفَذارى و برنامهريزى

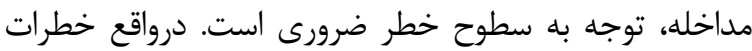

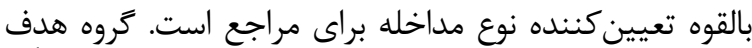

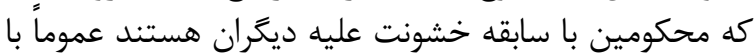

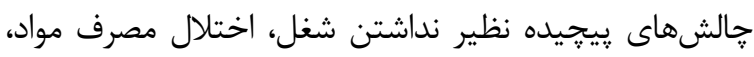

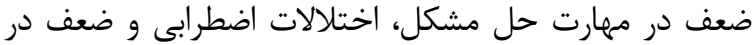

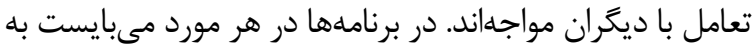

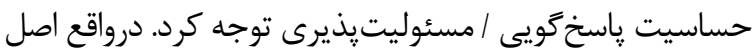

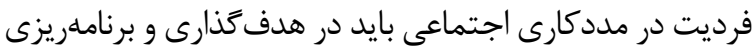

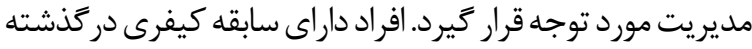

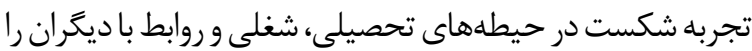

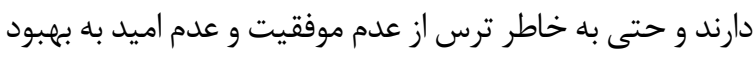

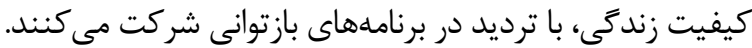

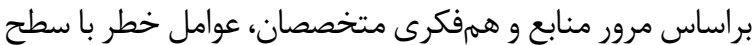

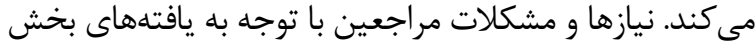

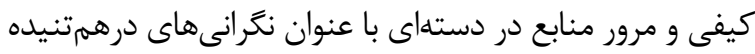

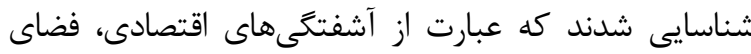

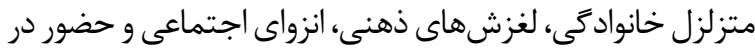

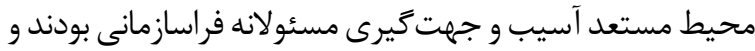

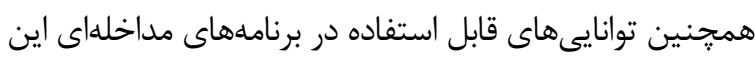

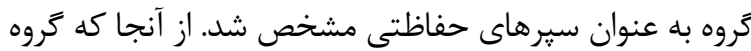

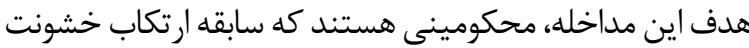

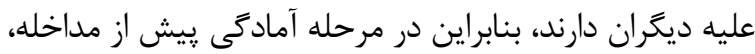

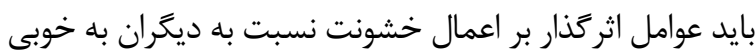
شناسايى شود و مبناى توسعه برنامه موردى قرار كيرد.

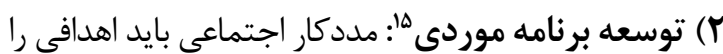

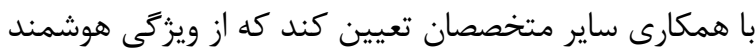

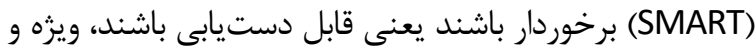

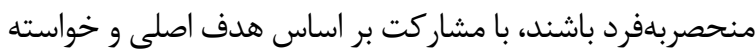

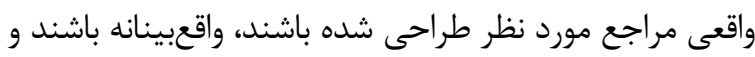

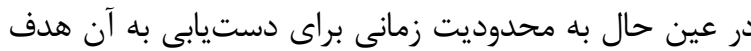

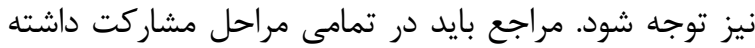

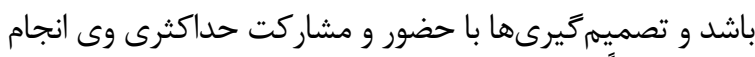

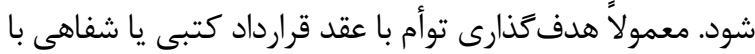

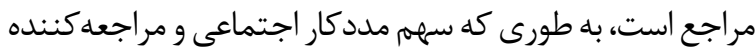

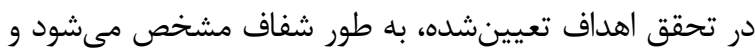

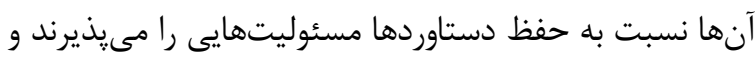

\section{Developing the case plan}


جدول ب. مخزن اوليه ابعاد بازكشت به جامعه افراد محكوم به خشويت عليه اشخاص (تركيب كار كيفى و مرور منابع) نمونهاي از كويهها / كدها

بازمّشت موفق به جامعه: ابعاد و

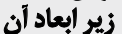

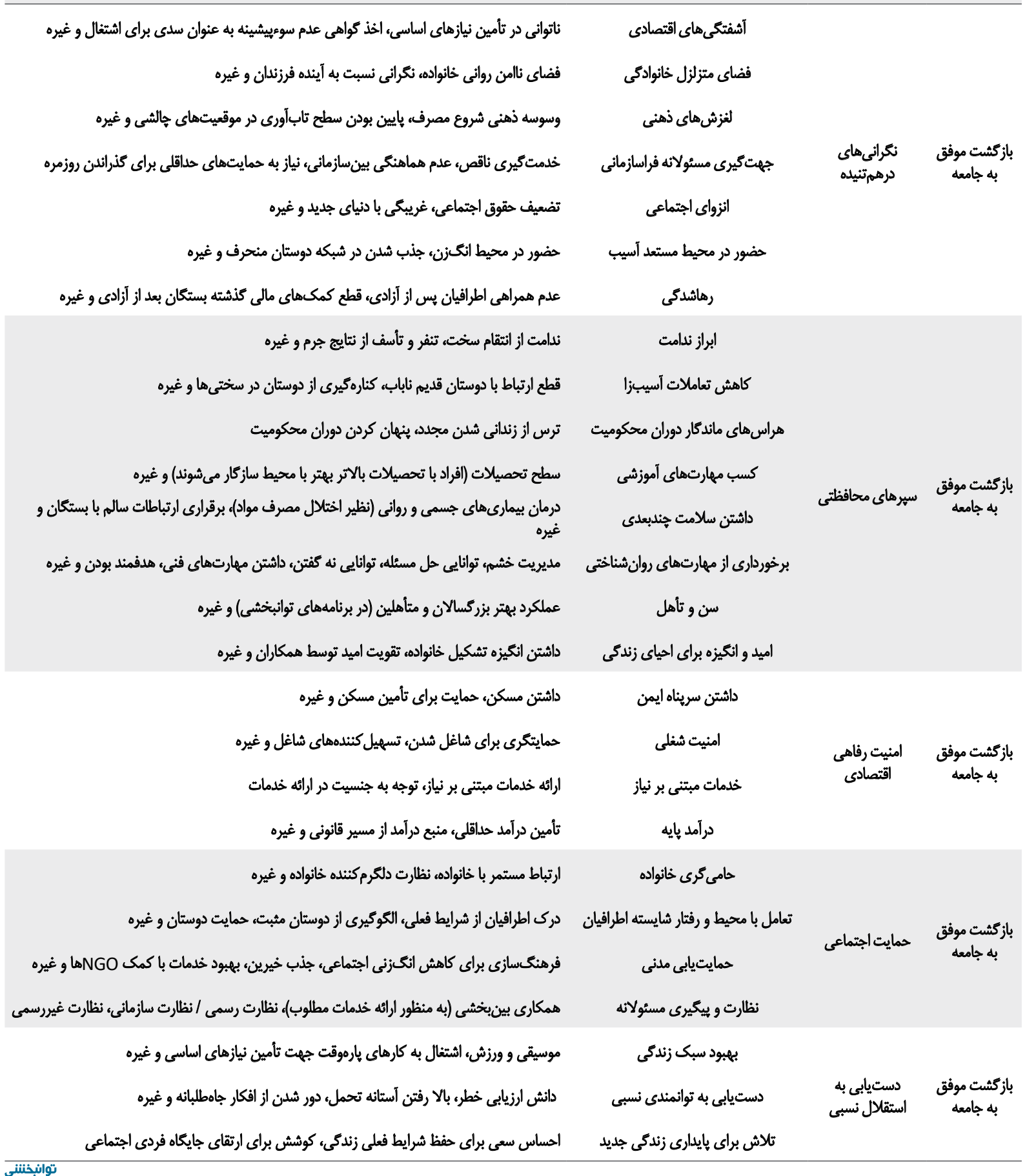

عنوان مثال، مراجعى كه مهارتى در نقاشى و معرق كارى دارد، اين

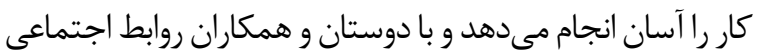

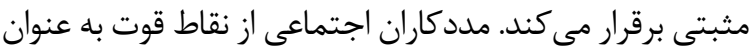

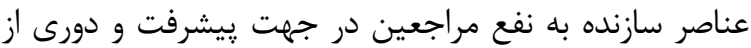
رفتار هاى مجرمانه كمك ميى
خطر مربوط به آن در جدول شماره ₹ ارائه شده است. طبق اين

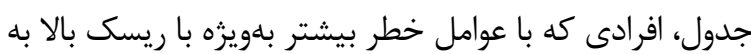

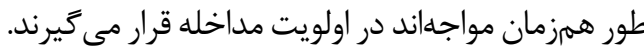
مدد كاران اجتماعى باشناسايى و استفاده از نقاط قوت مراجعين

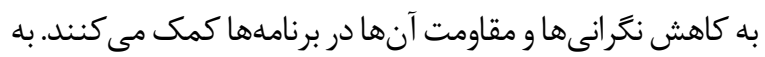


جدول "ا. مداخلات مبتنى بر رويكرد مديريت مورد

\begin{tabular}{|c|c|c|}
\hline دستاوردهاي مورد اتتظار & مداخلات و ابزارهاي مورد استفاده & كامهاى مداخله \\
\hline 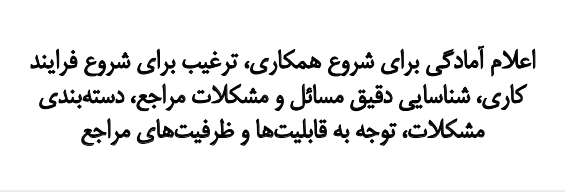 & 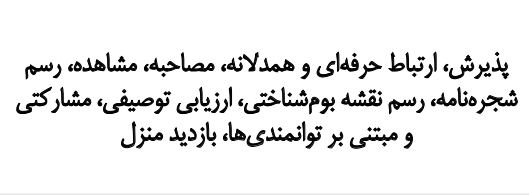 & 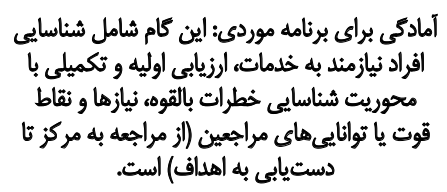 \\
\hline 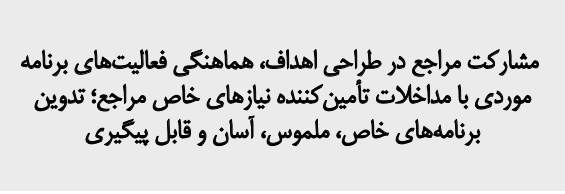 & 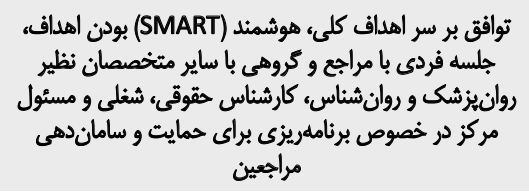 & توسعه برنامه موردى: اين مرحله شامل هدف كذارى \\
\hline شناسايع و الرزيابى مثنابع حمايتى، ييوند بين مراجع و منابع & 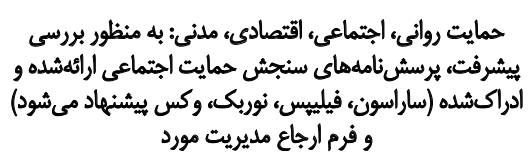 & \\
\hline 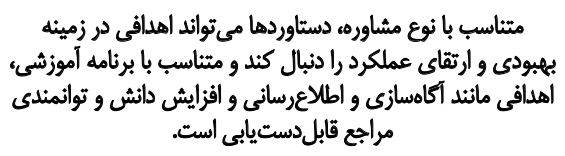 & 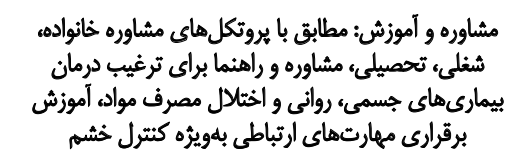 & \\
\hline 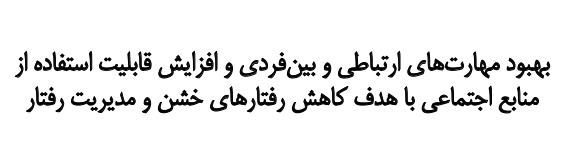 & 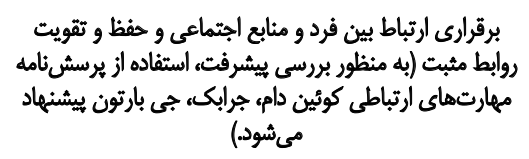 & \\
\hline بهبود مهارتهاى خانوادكى و وفع تششها، انجام تكليف در كروها، & 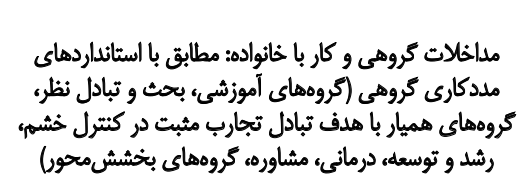 & 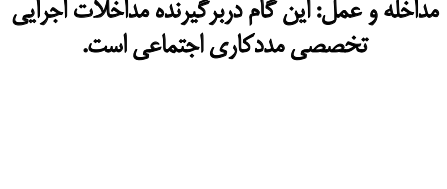 \\
\hline كاهش موانع الجتماعى، انكىزدايى، حمايتطلبى، حمايتكرى & 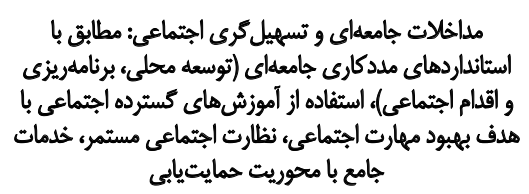 & \\
\hline 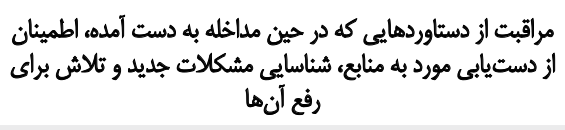 & 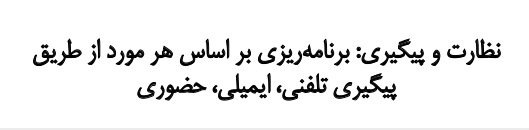 & \\
\hline 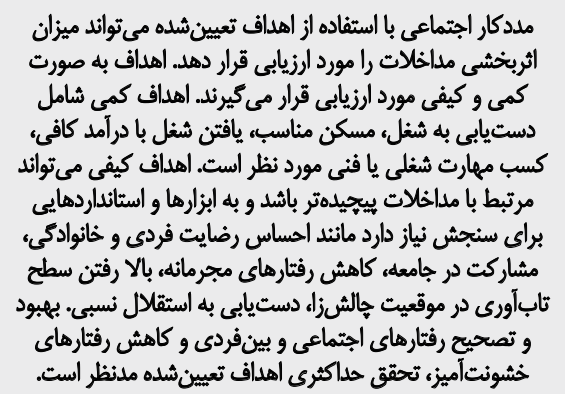 & 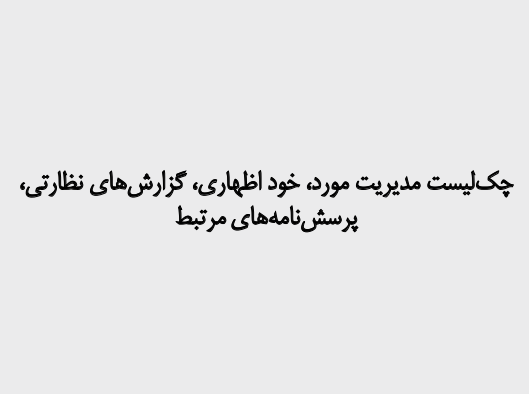 & 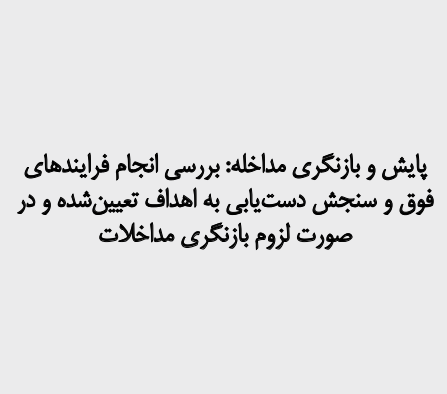 \\
\hline
\end{tabular}

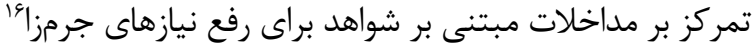

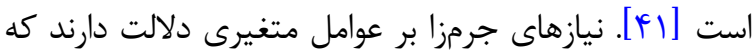

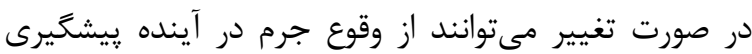
كنند؛ عواملى نظير نيازهاى اقتصادى رفاهى و روانى اجتماعى دري
مددكاران اجتماعى مىبايست از برنامههاى تحميلشده به

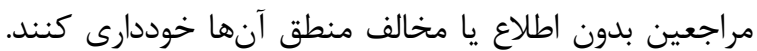

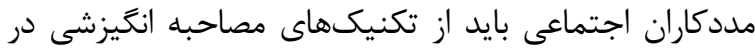

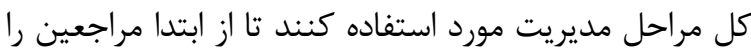

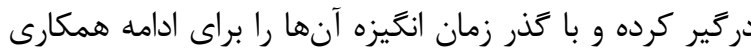

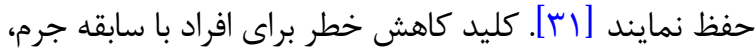


جدول ع. طبقهبندى مراجعين بر اساس ارزيابي ريسك

\begin{tabular}{|c|c|c|c|}
\hline باريسيك بالا (يرخطر) & باريسيك متوسط & باريسك بايين (كمخطر) & عوامل خحطر \\
\hline \multirow[t]{2}{*}{$*$} & & & ييشينه رفتارهاى خششونت آميز بيش ازئيكبار \\
\hline & * & & ضعف مهارتشاى ارتباطى \\
\hline \multirow[t]{3}{*}{$*$} & & & اعتياد به اللكل يا مواد مخلر \\
\hline & * & & بيكار \\
\hline & & * & مجرد \\
\hline$*$ & & & 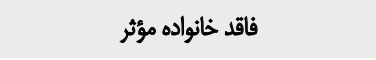 \\
\hline \multirow[t]{8}{*}{$*$} & & & طردشده از خاثواده \\
\hline & * & & سوءييشيئه بيش از يكبار \\
\hline & $*$ & & علم تمايل و اتكيزه براى تغيير \\
\hline & - & & نداشتن مسكن و سريناه مناسب \\
\hline & * & & امكان معاشرت با دوستان ناباب \\
\hline & & * & بايين بودن سطح تحصيلات \\
\hline & & * & نداشتن مهارت و أكاهى كافى \\
\hline & $*$ & & ابتلا به بيمارى هاى مقاريتى \\
\hline$*$ & & & سابقه ابثلا به بيمارى هاى روانى مزمن \\
\hline * & & & حبس هاى بلندملت بيش از ينج سال \\
\hline \multirow[t]{2}{*}{ * } & & & جرائم حُشن شديد نظير قتل \\
\hline & & $*$ & زندكى در محيط جرمزا / مستهد آسيب \\
\hline
\end{tabular}

"r) مداخله و عمل 'ا': مداخلات تخصصى با توجه به سطح خطرى كه مراجعين با آن مواجهاند و قضاوتهاى مداء حرفه مدد كارى اجتماعى شامل شش دسته هستند (تصوير شماره ()):

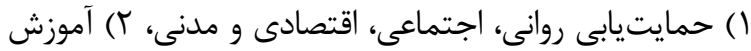

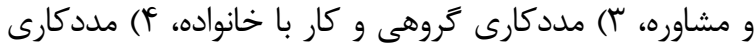
جامعهاى و تسهيل

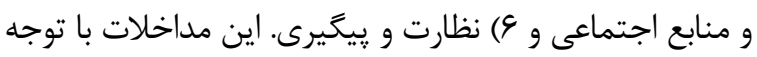

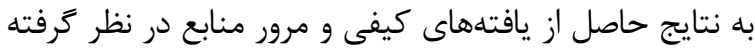

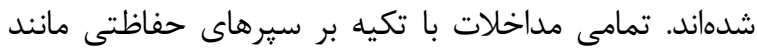

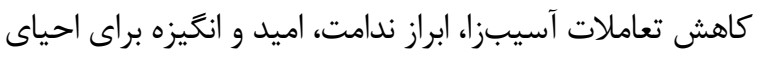

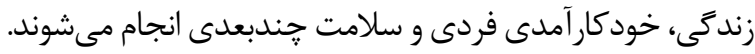

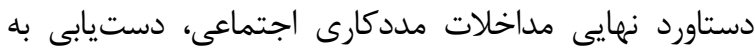

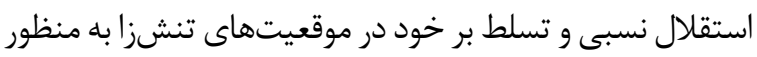
ييشخيرى از تكرار مجدد جرم و ييوند با جامعه است.

مديريت مورد مؤثر يك فرايند يوياست كه نياز به تعامل فعال
فرد بزهكار (نظير مسكن، شغل، تغذيه، اعتياد، عصبى بودن،

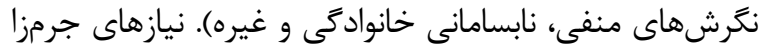
بيانكر سطح نارسايىهاى موجود هستند. نارسايى هايى كه گرجه

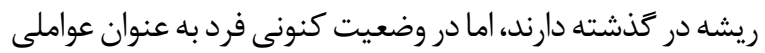

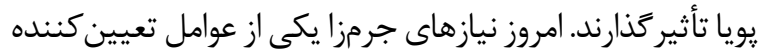

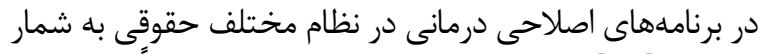

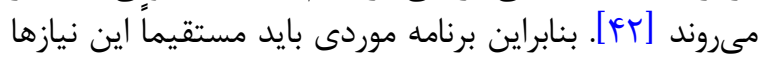

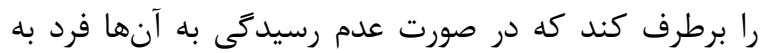

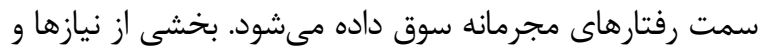

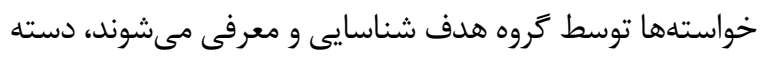

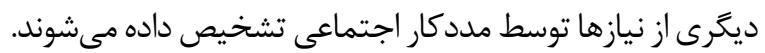

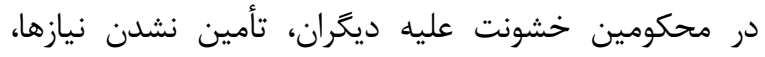

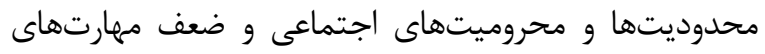

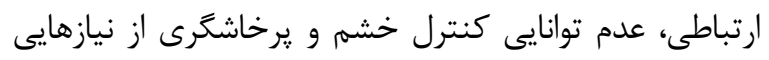

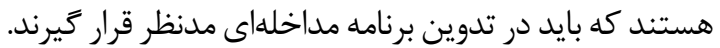




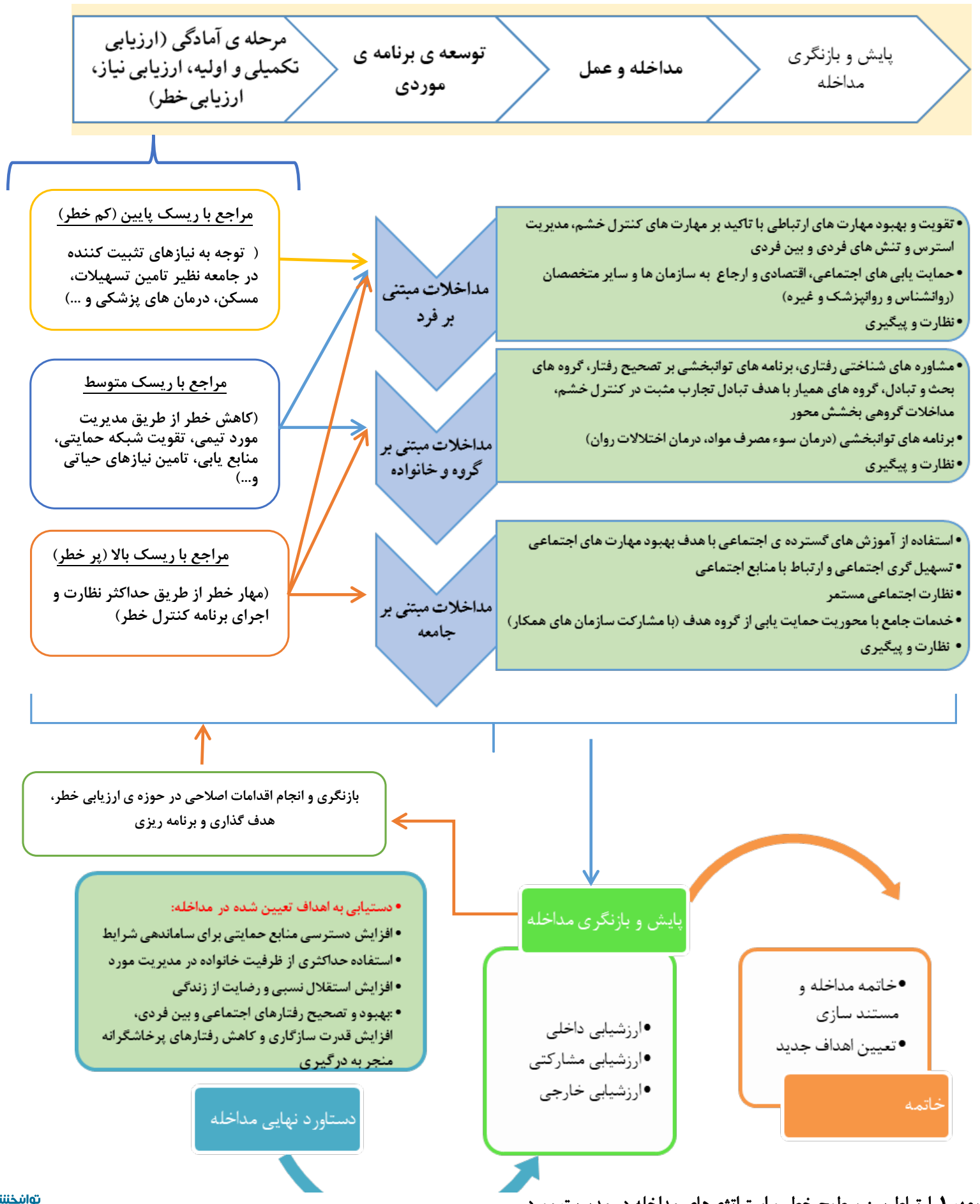

توانبخننى

تصوير ا. ارتباط بين سطوح خطر و استراترىهاى مداخله در مديريت مورد

كردن مراجعين كمخطر و يرخطر مىتواند يك اثر ياتروزنيك يا

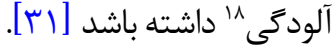

حوزهاى كه مددكاران اجتماعى مىبايست براى آن برنامهريزى

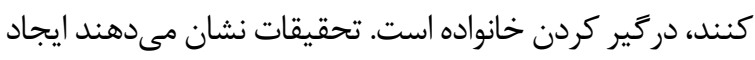
روابط قوى بين همان مجرمان سابق و خانوادههايشان استراتزى

18. latrogenic or contamination effect
و مداوم بين افراد و مددكاران اجتماعى و سازمان و اجتماع مداع

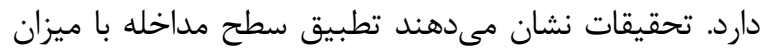

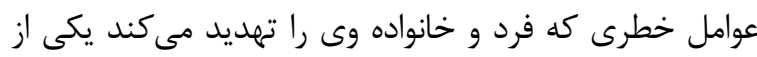

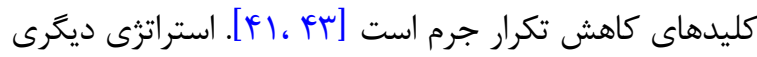

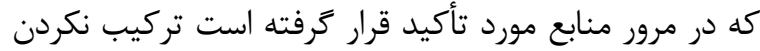

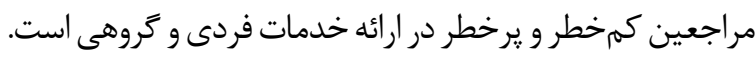

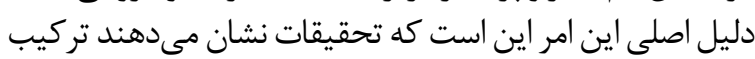


و تعهد به جامعه به حركت درمى آورد. بديهيهى است افر ادى كه در

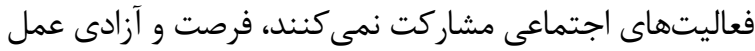

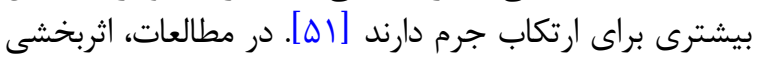

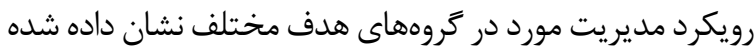

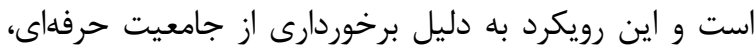

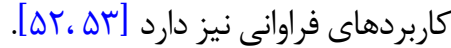

تحقيقات نشان مىدهند در رويكرد مديريت مورد، تطبيق

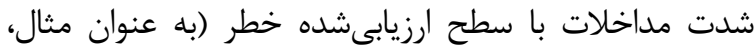

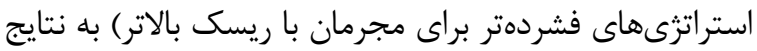

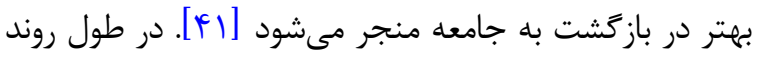

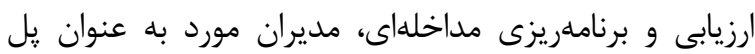

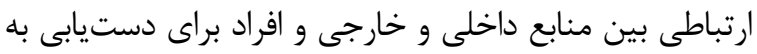

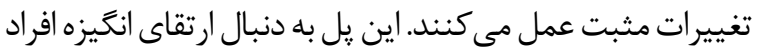

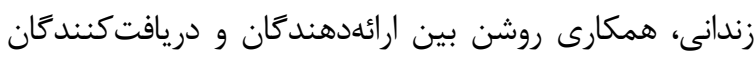

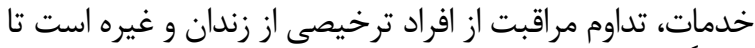

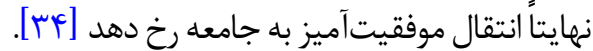
بر اساس فصل خدمات ادغام مجدد و نظارت يس إز آز آزادى

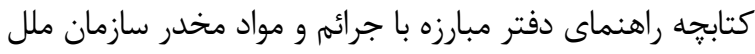

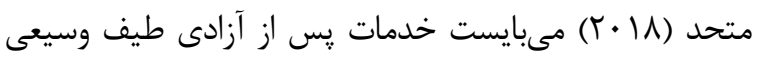

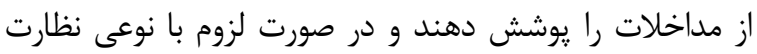

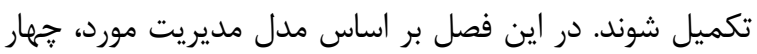

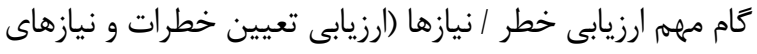

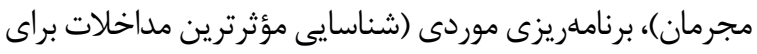

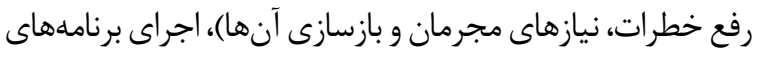

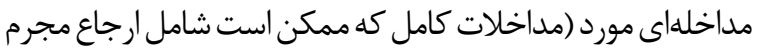

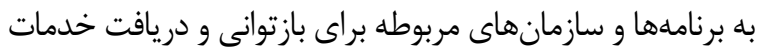

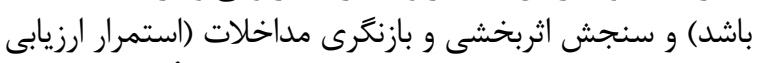

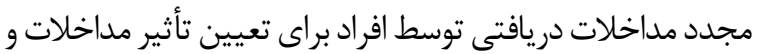

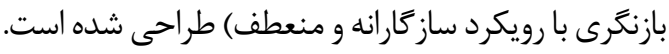

در منابع موجود همسو با يافتههاى اين مطالعه، يك راهنماى

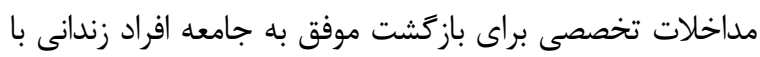

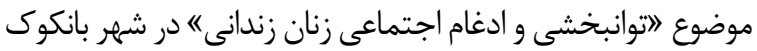

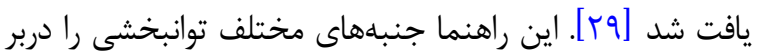

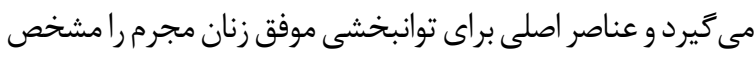

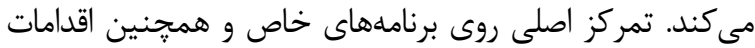

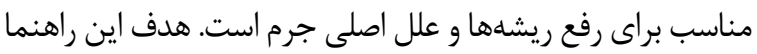

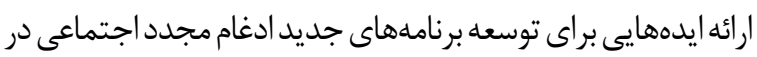

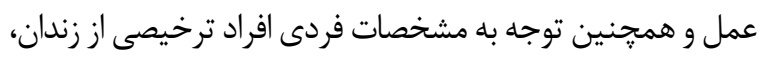

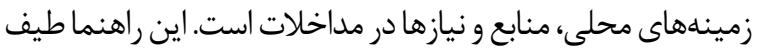

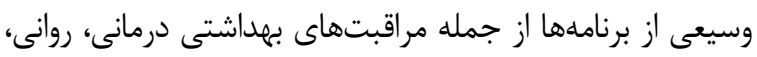

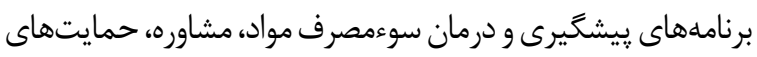

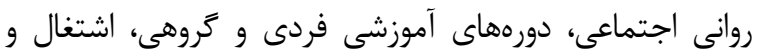

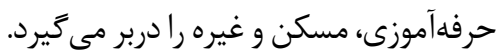

كارآمد براى بازَشت موفق به جامعه است. اين مشاركت به به

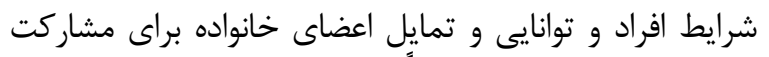

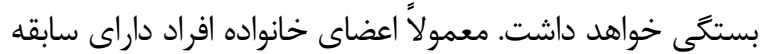

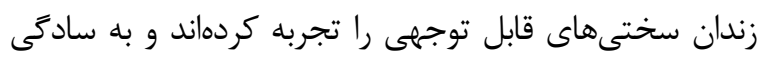

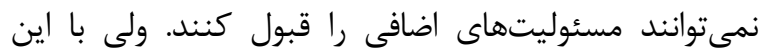

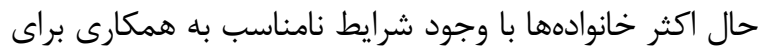

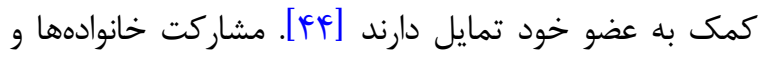

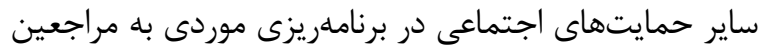

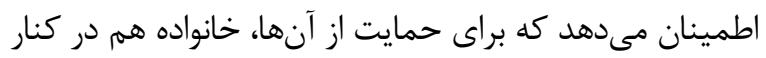

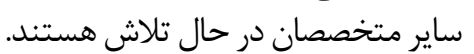

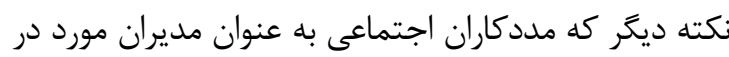

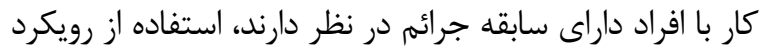

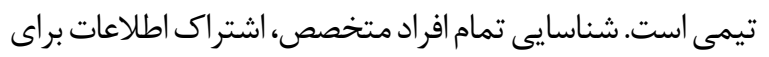

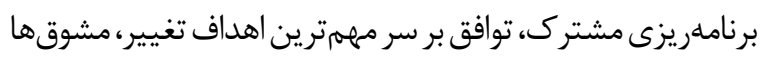

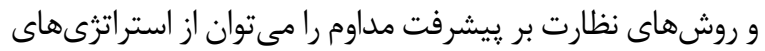
تأثيركذار مدير يت مورد قلمداد كرد.

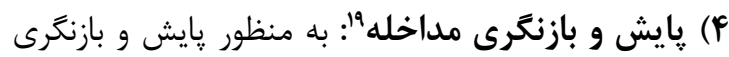

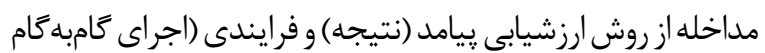

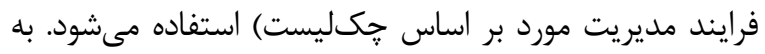

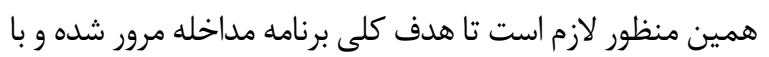

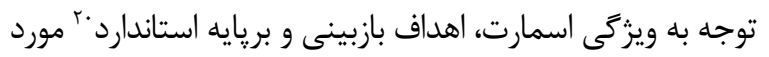

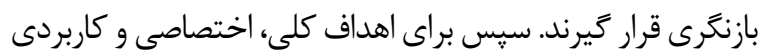

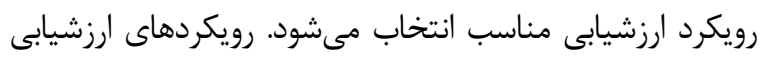

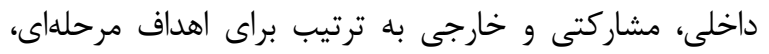

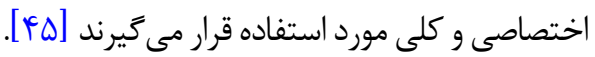

$\Leftrightarrow$

سرمايه كذارى در برنامههاى توانبخشى زندانيان يكى از بهترين

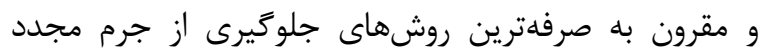

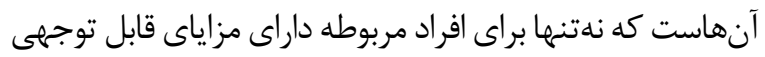

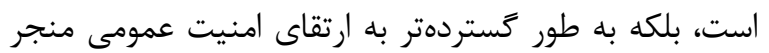

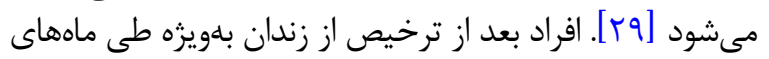

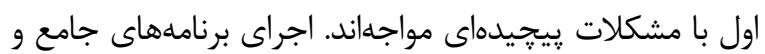

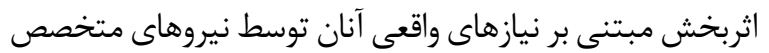

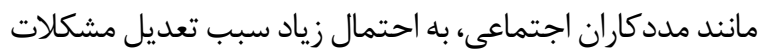

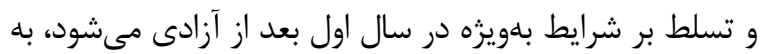

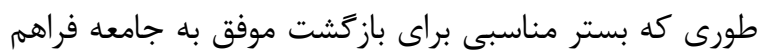

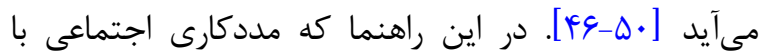

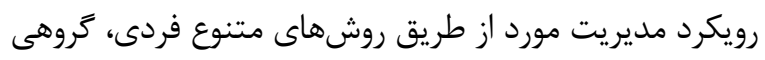

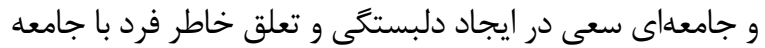

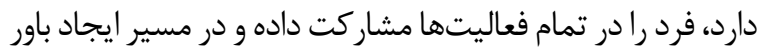

\section{Monitoring and reviewing the intervention} 20. Plan of action 
رويكرد مورد مديريت، حمايتيابى توسط سازمانهاى غيردولتى

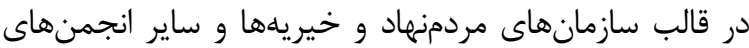

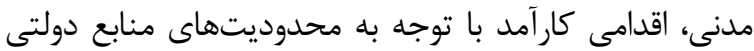

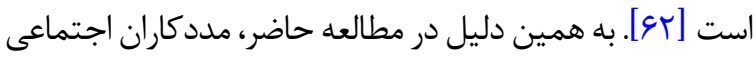

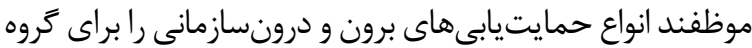

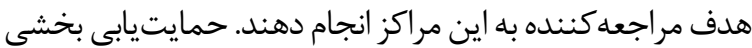

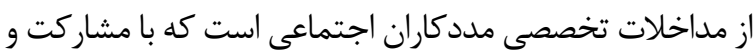

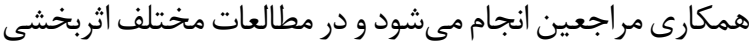

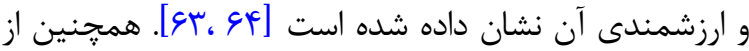

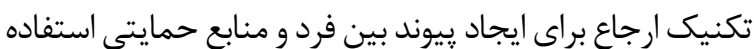

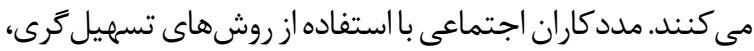

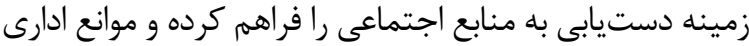

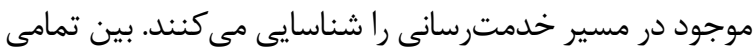

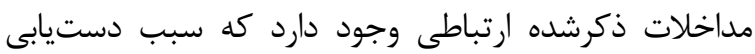

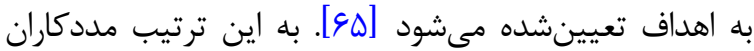

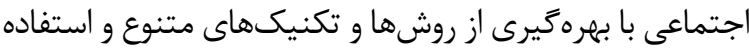

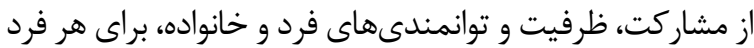

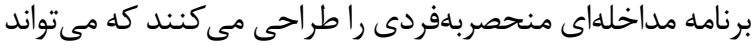

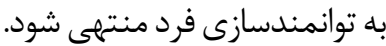

بلهور كلى، مددكاران اجتماعى با رويكرد مديريت مورد

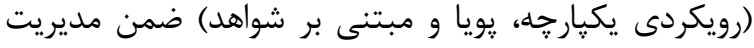

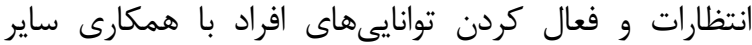

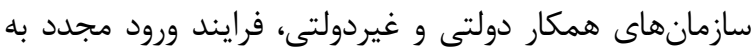

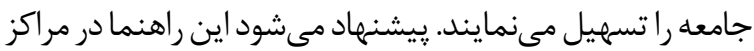
مراقبت بعد از خروج از زندان اجرا شود.

\section{نتيجلمَيرى}

خروجى نهايى اين مطالعه، توليد يك راهنماى مداخله مدد كارى

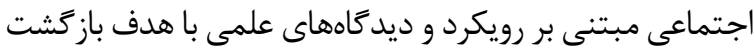

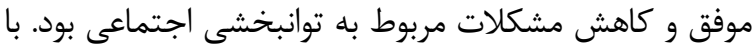

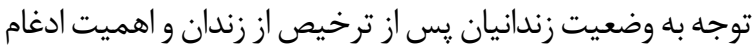

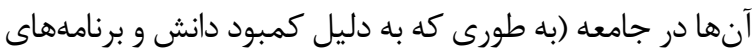

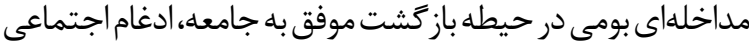

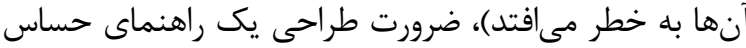

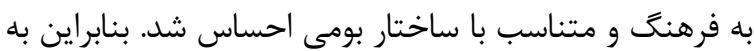

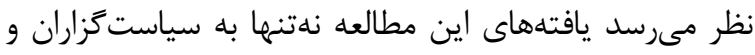

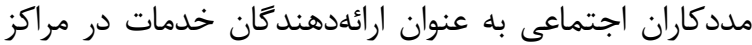

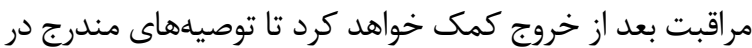

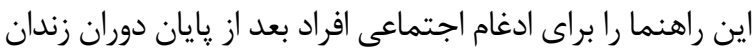

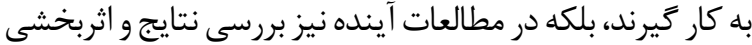

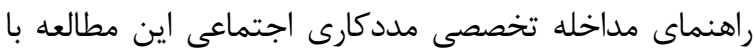

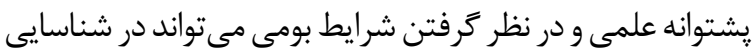

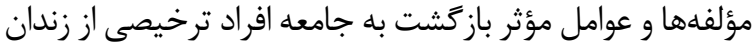

مطالعات گستردهاى نشان دادند ارتقاى توانمندىهاى روانى

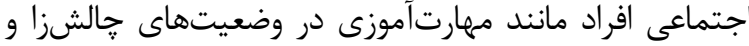

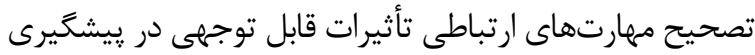

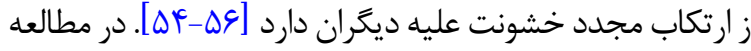

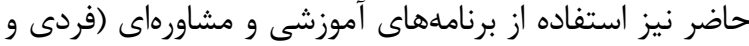

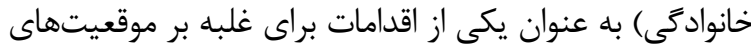

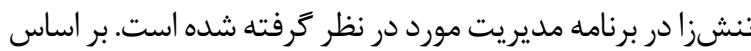

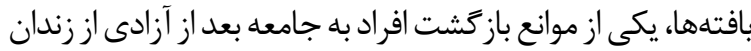

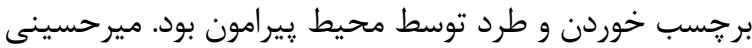

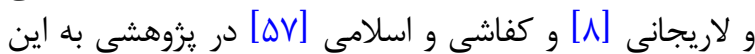

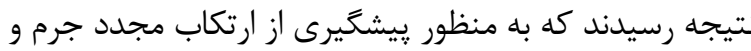

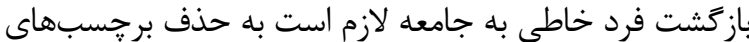

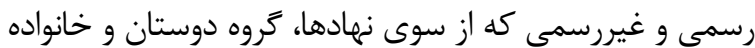

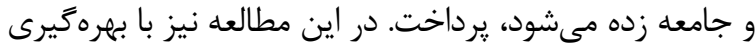

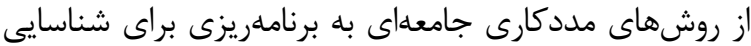

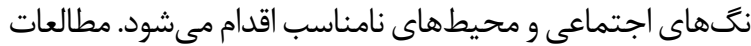

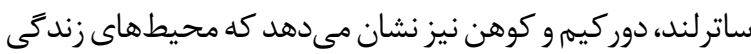

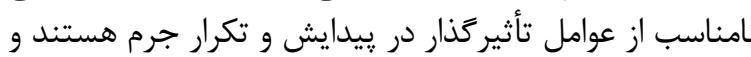

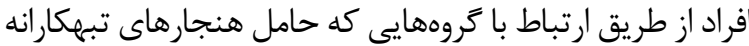

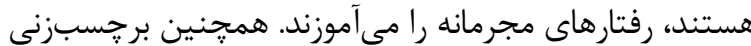

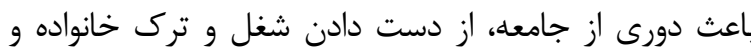

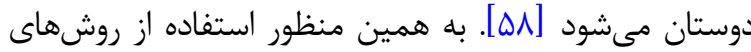

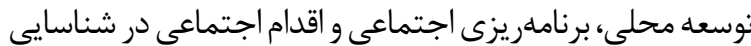

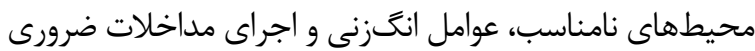

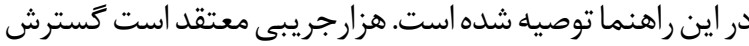

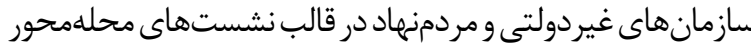

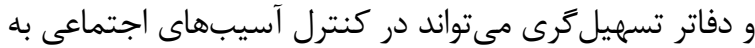
طور جامع مؤثر باشد [09]. حيطه ديخرى كه در اين راهنما بر آن تأكيد شد، حمايت

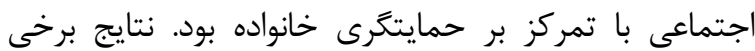

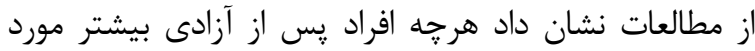

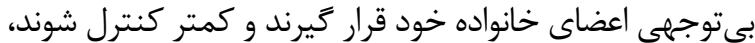

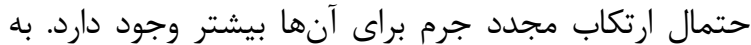

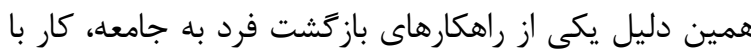

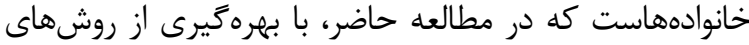

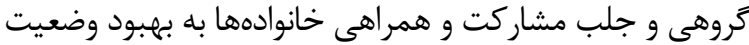

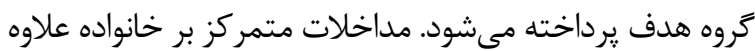

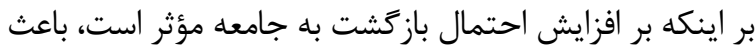

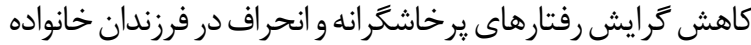

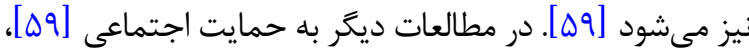

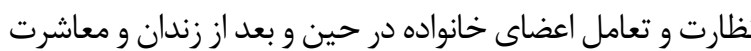

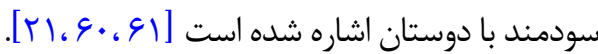
بنابراين استراتثى مهم در راهنماها و برنامههاى مداخله با 
بلويزه افراد محكوم به جرم خشونت عليه اشخاص مؤثر باشد.

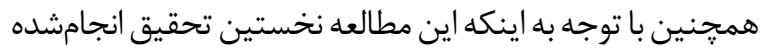

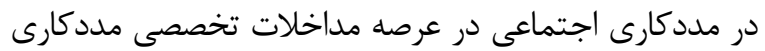

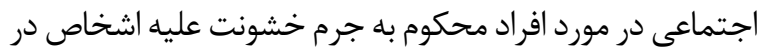

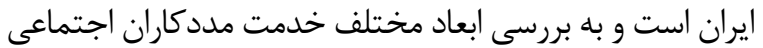

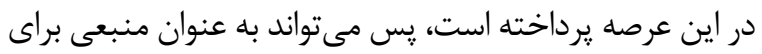
آموزش نيز مورد استفاده قرار كيرد.

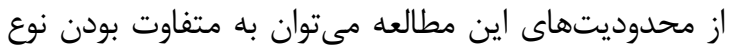

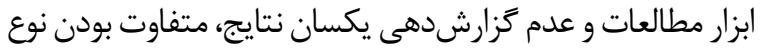

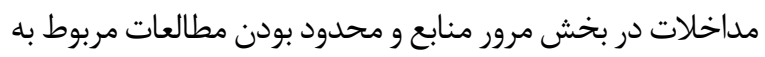
بازگشت به جامعه در كشور اشاره كرد.

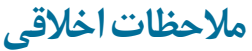

$$
\text { ييروى از اصول اخلاق يخوهش }
$$

توانئ اخلاقى يزوهش به تصويب كميته اخلاق دانشكاه علوم

توانبخشى و سلامت اجتماعى رسيده است (كد: IR.USWR.

(REC.1399.180

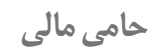

آين مقاله بخشى از رساله دكترى نويسنده اول در كروه

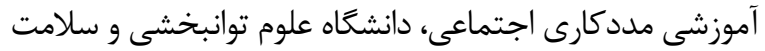

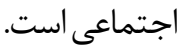

$$
\text { مشاركت نويسندكًان }
$$

همه نويسندكان در آمادهسازى اين مقاله به طور يكسان

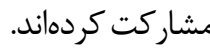

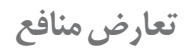

بنابر اظهار نويسندكان اين مقاله تعارض منافع ندارد.

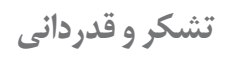

بدينوسيله از همكارى صميمانه اداره كل زندانهاى استان

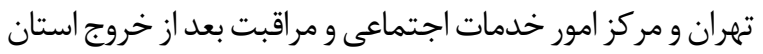

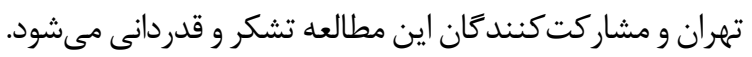




\section{References}

[1] Zeinali A. [Range of legislative intervention in the field of social problems and deviations (Persian)]. Social Welfare Quarterly. 2009; 9(34):301-20. http://refahj.uswr.ac.ir/article-1-2418-fa.html

[2] Asadolahi A, Baratvand M. [Study of relation between child abuse by parents and wickedness of their child (case study: Wickedness delinquents of Khuzestan) (Persian)]. Social Welfare Quarterly. 2006; 5(19):225-52. http://refahj.uswr.ac.ir/article-1-1956-en.html

[3] United Nations Office on Drugs and Crime. (2018).UNODC Annual Report 2018 [Internet]. 2018 [Updated 2018]. Available from: https://www.unodc.org/unodc/en/about-unodc/annual-report. $\mathrm{html}$

[4] Ahlin EM, Lobo Antunes MJ. Levels of guardianship in protecting youth against exposure to violence in the community. Youth Violence and Juvenile Justice. 2017; 15(1):62-83 [DOI:10.1177/1541204015590000]

[5] Zare B, Zandilak AA. [Level of socio-economic development and rates of violent crimes in the provinces (Persian)]. Journal of Iranian Social Studies. 2012; 5(3):97-125. http://www.jss-isa.ir/article_21453.htmlllang=en

[6] Iran Statistical Yearbook. No title [Internet]. 2018 Available from: https://www.amar.org.ir/Portals/1/yearbook/1397/15.pdf

[7] Vameghi M, Khodai Ardekani M, Sajadi H. [Domestic violence in Iran: Review of 2001-2008 literature (Persian)]. Social Welfare Quarterly. 2014; 13(50):37-70. http://refahj.uswr.ac.ir/article-1-1354-en. html

[8] Mirhosseini Z, Larijani M. [Identifying the context of designing a model for successful social integration of offender women: A grounded theory approach (Persian)]. Women in Development \& Politics. 2018; 16(1):51-78. [DOI:10.22059/JWDP.2018.246504.1007316]

[9] Cepeda JA, Vetrova MV, Lyubimova AI, Levina OS, Heimer R, Niccolai LM. Community reentry challenges after release from prison among people who inject drugs in St. Petersburg, Russia. International Journal of Prisoner Health. 2015; 11(3):183-92. [DOI:10.1108/ IJPH-03-2015-0007] [PMID] [PMCID]

[10] Choi J, Dulisse B. The Importation of violent "codes" of south korean inmates. The Prison Journal. 2020; 100(3):287-311. [DOI:10.1177/0032885520916816]

[11] Chikadzi V. Challenges facing ex-offenders when reintegration in to mainstream society in Gauteng South Africa. Social Work. 2017; 53(2):288-300. [DOI:10.15270/53-2-569]

[12] Sadeghi Fasaie S, Mirhosseini Z. [Life after prison: the challenges of reconstruction for women (Persian)]. Strategic Rssearch on Social Problems in Iran University of Isfahan. 2017; 6(3):31-50. [DOI:10.22108/SSOSS.2017.85536.0]

[13] Denney A, Tewksbury R, Jones R. Structure for successful offender reentry. Journal of Qualitative Criminal Justice \& Criminology. 2014 2(1):39-67. https://epublications.marquette.edu/socs_fac/96/

[14] Belenko S. Assessing released inmates for substance-abuse-related service needs. Crime and Delinquency. 2006; 52(1):94-113, [DOI:10.1177/0011128705281755]

[15] Freeman R. Can we close the revolving door?: Recidivism vs. employment of ex-offenders in the U.S. Symposium at the Urban Institute Reentry Roundtable, New York University Law School; 2003. [DOI:10.1037/e717762011-001]

[16] Mellow J, Christian J. Transitioning offenders to the community: A content analysis of reentry guides. Journal of Offender Rehabilitation. 2008; 47(4):339-55. [DOI:10.1080/10509670801992111]
[17] Wilson AJ, Davis RC. Good intentions meet hard realities: An evaluation of the project greenlight reentry program. Criminology and Public Policy. 2006; 5(2):303-38. [DOI:10.1111/j.17459133.2006.00380.x]

[18] Listwan SJ. Reentry for serious and violent offenders:an analysis of program attrition. Criminal Justice Policy Review. 2009; 20(2):154-69. [DOI:10.1177/0887403408325700]

[19] Travis J, Visher C. Prisoner reentry and crime in America. Cambridge: Cambridge University Press; 2005. [DOI:10.1017/ CBO9780511813580]

[20] Braun V, Clarke V. Using thematic analysis in psychology. Qualitative Research in Psychology. 2006; 3(2):77-101. [DOI:10.1191/1478088706qp063oa]

[21] Visher C. Incarcerated fathers: Pathways from prisonto home. Journal of Criminal Justice 2013; 24(1):9-26 [DOI:10.1177/0887403411418105]

[22] Miller HV, Miller JM. Community in-reach through jail reentry: findings from a quasi-experimental design. Justice Quarterly. 2010; 27(6):893-910. [DOI:10.1080/07418825.2010.482537]

[23] Petersilia J. When prisoners come home: Parole and prisoner reen try. New York: Oxford University Press; 2003. https://wwww.google. $\mathrm{com} /$ books/edition/_/cv Qc2dpL8AC?hl=en\&sa $=$ X\&ved=2ahU KEwiDovaC6Yr0AhVknVwKHbnKDQAQre8FegQIERAI

[24] Cnaan RA, Draine J, Fraizer B, Sinha JW. Ex-prisoners' re-entry: An emerging frontier and a social work challenge. Journal of Policy Practice. 2008. 7(2-3):178-98. [DOI:10.1080/15588740801938035]

[25] Toi H. Professional values and conflict among social workers in prisons: an examination of role stress, strain, and job satisfaction in working with inmates with mental illness and/or substance use disorders [PhD. Dissertation]. Mansfield: University of Connecticut; 2015. https://opencommons.uconn.edu/dissertations/992/

[26] Whittemore R, Knafl $\mathrm{K}$. The integrative review: Updated methodology. Journal of Advanced Nursing. 2005; 52(5):546-53 [DOI:10.1111/j.1365-2648.2005.03621.x] [PMID]

[27] Barnett-Page E, Thomas J. Methods for the synthesis of qualitative research: A critical review. BMC Medical Research Methodology. 2009; 9(59):1-11. [DOI:10.1186/1471-2288-9-59] [PMID] [PMCID]

[28] Chin V, Dandurand Y. Introductory handbook on the prevention of recidivism and the social reintegration of offenders. Criminal justice Handbook series [Internet]. 2018. https://www.unodc.org/ documents/justice-and-prison-reform/crimeprevention/Introductory_Handbook on the Prevention_of Recidivism and the Social_Reintegration_of_Offenders.pdf

[29] Crowley M. Guide to the rehabilitation and social reintegration of women prisoners [Internet]. 2019 [Updated 2019 May 13]. Available from: https://knowledge.tijthailand.org/en/publication/detail/ bangkok-rules\#book/

[30] Nancy GJ, Brazzell D. Cowan mapping prisoner reentry: An action research guidebook [Internet]. 2006. Available from: https://www ojp.gov/pdffiles1/niij/grants/213675.pdf

[31] Phyllis Modley, Center for Effective Public Policy and Rachelle Giguere, Center for Effective Public Policy. Reentry considerations for women offenders [Internet]. 2010. Available from: https://cepp. com/wp-content/uploads/2021/04/Reentry-Considerations-forWomen-Offenders-2010.pdf

[32] World Health Organization (WHO). WHO handbook for guideline development. Geneva: World Health Organization; 2014 https://apps.who.int/iris/handle/10665/145714 
[33] Brouwers MC, Kho ME, Browman GP, Burgers JS, Cluzeau F, Feder G, et al. AGREE II: Advancing guideline development, reporting and evaluation in health care. Canadian Medical Association Journal. 2010; 182(18):83-42. [DOI:10.1503/cmaj.090449] [PMID] [PMCID]

[34] Prendergast M, Cartier JJ. Improving parolees' participation in drug treatment and other services through strengths case management. Perspectives. 2008; 32(1):38-46. [PMID] [PMCID]

[35] Lipsey M. The primary factors that characterize effective interventions with juvenile offenders: A meta-analytic Overview. Victims \& Offenders. 2009; 4(2):124-47. [DOI:10.1080/15564880802612573]

[36] Willis GM, Ward T, Levenson JS. The Good Lives Model (GLM): An evaluation of GLM operationalization in North American treatment programs. Sexual Abuse. 2014; 26(1):58-81. [DOI:10.1177/1079063213478202] [PMID]

[37] Paparozzi MA, Gendreau P. An intensive supervision program that worked: Service delivery, professional orientation, and organizational supportiveness. The Prison Journal. 2005; 85(4):445-66.] [DOI:10.1177/0032885505281529]

[38] Lattimore PK, Visher CA. The impact of prison reentry services on short-term outcomes: Evidence from a multisite evaluation. Evaluation Review. 2013; 37(3-4):274-313. [DOI:10.1177/0193841X13519105] [PMID]

[39] Osher F, Steadman HJ, Barr H. A best practice approach to community reentry from jails for inmates with co-occurring disorders: The apic model. Crime \& Delinquency. 2003; 49(1):79-96. [DOI:10.1177/0011128702239237]

[40] Hunter BA, Lanza AS, Lawlor M, Dyson W, Gordon DM. A strengths-based approach to prisoner reentry: The fresh start prisoner reentry program. International Journal of Offender Therapy and Comparative Criminology. 2016; 60(11):1298-314. [DOI:10.1177/0306624X15576501] [PMID] [PMCID]

[41] Andrews D, Dowden C. The risk-need-responsivity model of assessment and human service in prevention and corrections: crime-prevention jurisprudence. Canadian Journal of Criminology and Criminal Justice. 2007; 49(4):439-64. [DOI:10.3138/ cjсcj.49.4.439]

[42] Ashouri M, Ghasemi Moghadam H. [Methods of actuarial crime risk assessment (Persian)]. Private Low Studies Quarterly. 2014; 43(4):1-18. [DOI:10.22059/JLQ.2014.50102]

[43] Andrews DA, Bonta J, Wormith JS. The recent past and near future of risk and/or need assessment. Crime \& Delinquency. 2006; 52(1):7-27. [DOI:10.1177/0011128705281756]

[44] Niven S, Stewart D. Resettlement outcomes on release from prison in 2003 [Internet]. 2005 [Updated 2005]. Available from: https://lemosandcrane.co.uk/resources/Home $\% 20$ Office $\% 20248 \% 20-\% 20$ Resettlement $\% 20$ outcomes $\% 20$ on $\% 20$ release $\% 20$ from $\% 20$ prison. pdf

[45] Nasirzadeh M. The process of designing, implementation and evaluation of theory-based educational interventions. Journal of Rafsanjan University of Medical Sciences. 2020; 19(5):549-52. [DOI:10.29252/jrums.19.5.549]
[46] Turner S, Perersilia J. Work Release in Washington: Effects on recidivism and corrections costs. The Prison Journal. 1996; 76(2):138-64. [DOI:10.1177/0032855596076002003]

[47] Ruch DA, Yoder JR. The effects of family contact on community reentry plans among incarcerated youths. Victims \& Offenders. 2018; 13(5):609-27. [DOI:10.1080/15564886.2017.1401571]

[48] Bouffard JA, Bergseth KJ. The impact of reentry services on juvenile offenders' recidivism. Youth Violence and Juvenile Justice. 2008; 6(3):295-318. [DOI:10.1177/1541204007313384]

[49] Shivy VA, Guion DB, Green BA, Wingate JA. INTUIT: A career decision-making intervention for female offenders in reentry. Journal of Career Development. 2019; 46(2):144-56. [DOI:10.1177/0894845317731156]

[50] Garland B, Wodahl E, Saxon C. What influences public support of transitional housing facilities for offenders during reentry? Criminal Justice Policy Review. 2017; 28(1):18-40. [DOI:10.1177/0887403414564866]

[51] Ramakers A, Nieuwbeerta P, Van Wilsem J, Dirkzwager A. Not just any job will do: A study on employment characteristics and recidivism risks after release. International Journal of Offender Therapy and Comparative Criminology. 2017; 61(16):1795-818. [DOI:10.1177/0306624X16636141] [PMID] [PMCID]

[52] Rapp RC, Van Den Noortgate W, Broekaert E, Vanderplasschen W. The efficacy of case management with persons who have substance abuse problems: A three-level meta-analysis of outcomes. Journal of Consulting and Clinical Psychology. 2014; 82(4):605-18. [DOI:10.1037/a0036750] [PMID]

[53] Solomon P. The efficacy of case management services for severely mentally disabled clients. Community Mental Health Journal. 1992; 28(3):163-80. [DOI:10.1007/BF00756815] [PMID]

[54] Rasouli F. [The impact of anger control training on resiliency in adolescents with addiction preparation (Persian)]. Scientific Quarterly of Research on Addiction. 2011; 4(16):7-24. http://etiadpajohi. ir/article-1-366-en.html

[55] Sadeghi S, Farajzadegan Z, Kelishadi R, Heidari K. Aggression and violence among Iranian adolescents and youth: A 10-year systematic review. International Journal of Preventive Medicine. 2014; 5(2):8396. [DOI:10.4103/2008-7802.157663] [PMID]

[56] Roustai A, Abolghasemi S, Mohammadi Arya A, Saedi S. [A comparison effect of training the anger management and medicine cure on aggression in prisoners (Persian)]. Journal of Social Psychology. 2011; 7(21):19-31. https://www.sid.ir/fa/Journal/ViewPaper. aspx?id $=160120$

[57] Kafashi, M., Eslami, E. The study of effective factors on reoffending and reentry to prison in the province of Northern Khorasan (Persian)]. Journal of Social Reserch. 2009; 2(4):57-69. https://www.sid.ir/en/Journal/ViewPaper.aspx?ID=181879

[58] Hezar Jaribi J, Safari Shali R. [Factors affecting the commitment of crimes (Case study: Markazi province prisoners) (Persian)]. Social Sciences. 2009; 16(46):41-74. https://qjss.atu.ac.ir/article_5383.html?lang=en

[59] Hochstetler A, DeLisi M, Pratt TC. Social support and feelings of hostility among released inmates. Crime \& Delinquency. 2010; 56(4):588-607. [DOI:10.1177/0011128708319926] 
[60] Kelso KT. A family affair: The effects of familial relations on offender recidivism [PhD. dissertation]. Detroit: Wayne State University; 2012. https://www.proquest.com/openview/9b17b0d0d c7c1b8fe12ed102ead08287/1?pq-origsite $=$ gscholar\&cbl $=18750$

[61] Léger A. Restoration or retribution: An empricial examination of the recidivistic patterns of a group of young offenders from new York city [MA. thesis]. Ontario: Queen's University; 2009. https:// qspace.library.queensu.ca/handle/1974/5364

[62] Ramsden V, Martin R, McMillan J, Granger-Brown A, Tole B. Participatory health research within a prison setting: A qualitative analysis of 'Paragraphs of passion. Global Health Promotion 2015; 22(4):48-55. [DOI:10.1177/1757975914547922] [PMID]

[63] McNutt J. Is social work advocacy worth the cost? Issues and barriers to an economic analysis of social work political practice. Research on Social Work Practice. 2011; 21(4):397-40. [DOI:10.1177/1049731510386624]

[64] Dalrymple J, Boylan J. Effective advocacy in social work. New York: SAGE; 2015. https://www.google.com/books/edition/_/ QsmYAQAACAAJ?hl=en\&sa=X\&ved $=2$ ahUKEwisgJPOpov0 AhUvz4UKHT7eB9UQre8FegQIDhAJ

[65] Ronen T. Collaboration on critical questions in child psychotherapy: A model linking referral, assessment, intervention, and evaluation. Journal of Social Work Education. 2001; 37(1):91-110. [DOI:10.1080/10437797.2001.10779039] 
This Page Intentionally Left Blank 